\title{
BPS/CFT CORRESPONDENCE IV: SIGMA MODELS AND DEFECTS IN GAUGE THEORY
}

\author{
Nikita Nekrasov*
}

\begin{abstract}
Quantum field theory $L_{1}$ on spacetime $X_{1}$ can be coupled to another quantum field theory $L_{2}$ on a spacetime $X_{2}$ via the third quantum field theory $L_{12}$ living on $X_{12}=X_{1} \cap X_{2}$. We explore several such constructions with two and four dimensional $X_{1}, X_{2}$ 's and zero and two dimensional $X_{12}$ 's, in the context of $\mathcal{N}=2$ supersymmetry, non-perturbative Dyson-Schwinger equations, and BPS/CFT correspondence. The companion paper 99. will show that the BPZ and $\mathrm{KZ}$ equations of two dimensional conformal field theory are obeyed by the half-BPS surface defects in quiver $\mathcal{N}=2$ gauge theories.
\end{abstract}

\section{Introduction}

Quantum field theory, with all its successes in describing the world of elementary particles, still lacks proper mathematically rigorous definition. One of the actively discussed topics is the locality of the space of states of the theory, how entangled are the states, and, more generally, the spatial distribution of the elementary building blocks of the finite energy states of the theory. In recent years the concept of generalized symmetries, acting between different quantum field theories, has been emerging. A possible way of realizing such symmetries is by merging two quantum field theories across a defect, as discussed in [101] and, less explicitly, in the last section of [106].

Given two Euclidean Lagrangian quantum field theories: one with some fields $\boldsymbol{\Phi}_{1}$ defined on a spacetime manifold $X_{1}$ and the action $L_{1}\left(\boldsymbol{\Phi}_{1}\right)$, and another with some fields $\boldsymbol{\Phi}_{2}$ defined on a spacetime manifold $X_{2}$ and the action $L_{2}\left(\boldsymbol{\Phi}_{2}\right)$, we can couple them along the intersection $X_{12}=X_{1} \cap X_{2}$ assuming it is non-empty. For example, suppose another set of fields $\boldsymbol{\Psi}$ live on $X_{12}$ only, with the Lagrangian $L_{12}\left(\Psi ;\left.\boldsymbol{\Phi}_{1}\right|_{X_{12}},\left.\boldsymbol{\Phi}_{2}\right|_{X_{12}}\right)$ containing the couplings to the restrictions of the "bulk" fields onto the intersection.

\footnotetext{
*Simons Center for Geometry and Physics, Stony Brook University, Stony Brook, NY 11794 , on leave of absence from: Kharkevich IITP RAS, Moscow, ITEP, Moscow .

e-mail:nnekrasov@scgp.stonybrook.edu
} 


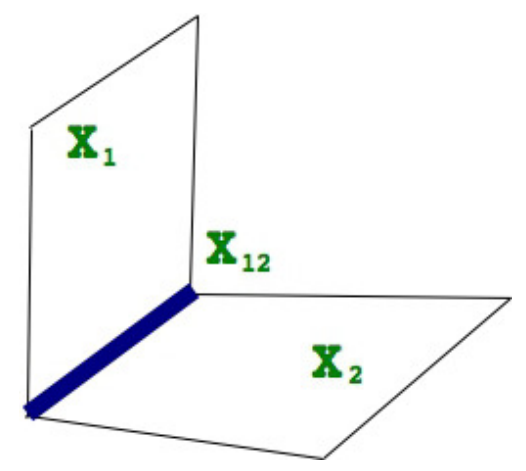

The simplest such example can be found in quantum mechanics, see appendix A.

While the maps (morphisms) between two quantum field theories come from the codimension one defects, the categorification suggests the studies of defects of all possible codimensions. In this paper we shall be mostly discussing the codimension two defects.

The paper is organized as follows. The section 2 recalls and clarifies some of the wellknown facts about the two dimensional gauge theories and their point-like defects, as well as their three dimensional lifts and line defects. In particular, we discuss the moduli spaces of flat connections on punctured Riemann surfaces, and relate integration over these spaces to supersymmetric two dimensional Yang-Mills theory with point-like defects. The section 3 adds matter to these theories to make them gauged sigma models, then embeds these theories as a specific low-energy limit of compactified higher dimensional gauge theories. The sigma models on the moduli spaces of flat connections on punctured surfaces, in this context, become the four dimensional gauge theories with surface defects. The section 4 introduces the supersymmetric partition functions, defined as a limit of twisted Witten index of a higher dimensional theory. We discuss equivariant index of Dirac operator on the moduli spaces of solutions to generalized Hitchin equations, quiver theories, and specific examples of sigma models on Grassmanians and vector bundles over them. The section 5 reviews a noncommutative gauge theory approach to the same problems. One views the gauge theory on a noncommutative space as a deformation of the original theory. The supersymmetric partition function has no continuous dependence on the noncommutativity parameters, allowing one to reduce the computation to a combinatorial formula. The section $\mathbf{6}$ studies in detail three constructions of surface defects: the orbifold defects, the quiver defects, and the folded defects. The section $\mathbf{7}$ discusses a specific class of local operators in two dimensional gauged linear sigma models, the $q 9$-characters, which can also be viewed as a particular case of the codimension two defects. These operators are useful in revealing the hidden symmetries of quantum field theories we are studying. The concluding section $\mathbf{8}$ discusses the topics we left out.

\section{Examples in two dimensions}

Consider the two dimensional Yang-Mills theory with the gauge group $G$, a simple Lie group. In the cohomological field theory formulation is has the fields $A, \psi, \sigma$ with $A$ a connection, $\psi$ an adjoint-valued fermion one form, and $\sigma$ and adjoint scalar, the electric field of the first order formalism. One assigns a $U(1)$-charge (ghost number) to these fields, with $A$ being neutral, $\psi$ of charge 1 and $\sigma$ of charge 2 . The action is (we denote by Tr the Killing form on 
$\mathfrak{g}=\operatorname{Lie}(G))$

$$
S_{Y M}=-\frac{\mathrm{i} t}{4 \pi^{2}} \int_{\Sigma} \operatorname{Tr}\left(\sigma F_{A}+\frac{1}{2} \psi \wedge \psi\right)+\frac{u}{8 \pi^{2}} \int_{\Sigma} \operatorname{Tr} \sigma^{2} \operatorname{vol}_{\Sigma}
$$

where

$$
\frac{u}{t^{2}}=g_{\mathrm{ym}}^{2}
$$

is the Yang-Mills coupling constant. The theory (1) has the fermionic symmetry, of ghost number 1:

$$
\delta A=\psi, \quad \delta \psi=d_{A} \sigma, \quad \delta \sigma=0
$$

representing the equivariant de Rham differential in the Cartan model of the equivariant cohomology of the space of gauge fields, with the symmetry group being the group of gauge transformations. The cohomology of $\delta$ in the space of local operators is isomorphic to the ring of $G$-invariant polynomials on the Lie algebra $\mathfrak{g}=\operatorname{Lie}(G)$, represented by the operators

$$
\mathcal{O}_{P}^{(0)}(x)=P(\sigma(x))
$$

The $\delta$-symmetry defines the so-called descent procedure,

$$
\delta \mathcal{O}_{P}^{(i)}=d \mathcal{O}_{P}^{(i-1)}
$$

which associates to every local operator (4) and a homology class $\alpha \in H_{i}(\Sigma, \mathbb{Z})$ the $\delta$-closed observables

$$
\int_{\alpha} \mathcal{O}_{P}^{(i)}, \quad i=0,1,2
$$

which we shall call the integrated $i$-observables. The action (1) is actually a sum of a twoobservable and a 'smeared' zero-observable. The operators Tr $\sigma^{n}$ have the ghost number $2 n$, the $i$-observable $\mathcal{O}_{\operatorname{Tr} \sigma^{n}}^{(i)}$ have the ghost number $2 n-i$.

When $\Sigma=\Sigma_{g}$ is a genus $g$ compact Riemann surface, the partition function of the theory (1) , up to the nonperturbative in $g_{\text {ym }}^{2}$ terms, would be equal to:

$$
Z(t, u) \sim \int_{\mathcal{M}_{g}(G)} e^{t \omega+u \Theta}=\sum_{k=0}^{[d / 2]} \frac{t^{d-2 k} u^{k}}{k !(d-2 k) !} \int_{\mathcal{M}_{g}(G)} \omega^{d-2 k} \wedge \Theta^{k},
$$

the integral over the moduli space $\mathcal{M}_{g}(G)$ of flat $G$-connections on $\Sigma_{g}$, of a product of the symplectic form $\omega$ and a certain Pontryagin class $\Theta[120$. The $U(1)$ ghost number symmetry has a gravitational anomaly, so that on the worldsheet $\sum$ only the operators of the total ghost number $2 d \equiv-\operatorname{dim}(G) \chi(\Sigma)$ may have the non-zero expectation value. The ghost number anomaly coincides with the dimension of the moduli space of flat connections

$$
\operatorname{dim} \mathcal{M}_{g}(G)=-\chi\left(\Sigma_{g}\right) \operatorname{dim}(G),
$$

where

$$
\mathcal{M}_{g}(G)=\operatorname{Hom}\left(\pi_{1}\left(\Sigma_{g}\right), G\right) / G
$$

Unfortunately, the moduli space is not smooth (unless one turns on a discrete 't Hooft flux), so that the path integral actually localizes onto the integral over the non-compact singular space of solutions to the equations

$$
F_{A}=0, \quad d_{A} \sigma=0
$$


making the $u$-dependence of $Z(t, u)$ non-analytic.

We shall now couple the two-dimensional gauge theory to a zero-dimensional one. The latter is a theory of integrals over the spaces with $G$-symmetry. The building blocks of such spaces, in some sense, are the coadjoint orbits. Let $T \subset G$ be the maximal torus, and $\mathrm{t}=\operatorname{Lie}(T) \subset \mathfrak{g}$ the Cartan subalgebra. For $v \in \mathfrak{g}^{*}$ let $O_{v} \subset \mathfrak{g}^{*}$ denote the coadjoint orbit:

$$
O_{v}=\left\{A d_{g}^{*}(v) \mid g \in G\right\}
$$

Let $\omega_{v} \in \Omega^{2}\left(O_{v}\right)$ denote Kirillov-Kostant-Souriau form, the canonical symplectic form on $O_{v}$, and

$$
\mu_{v}: O_{v} \longrightarrow \mathfrak{g}^{*}
$$

the moment map, which coincides with the embedding map. Finally, let

$$
\mathcal{V}_{v}(\sigma)=\int_{O_{v}} e^{\varrho_{v}+\mathrm{i} \mu_{v}(\sigma)}
$$

be the equivariant symplectic volume of $O_{v}$, an $A d$-invariant function of $\sigma \in \mathfrak{g}$. One can compute $\mathcal{V}_{v}(\sigma)$ rather explicitly. Choose a representative of $\sigma$ in the Cartan subalgebra. Let us denote it by the same letter. For the generic $v, O_{v} \approx G / T$, and generic $\sigma \in \mathrm{t}$ :

$$
\mathcal{V}_{v}(\sigma)=\frac{1}{\prod_{\alpha>0} \alpha(\sigma)} \sum_{w \in W}(-1)^{w} e^{\mathrm{i} v\left(\sigma^{w}\right)}
$$

where $\alpha \in \mathrm{t}^{*}$ are the roots of $\mathfrak{g}, W$ is the Weyl group of $G$, and $\sigma^{w}$ is the $w$-transformed $\sigma$. For example, for $G=S U(n), T \approx U(1)^{n-1}, W=S(n)$, the symmetric group, $v$ and $\sigma$ are diagonal matrices, and the condition that $v$ is generic means that the eigenvalues $v_{i}, i=1, \ldots, n$ of $v$ are distinct. If, in addition, $\sigma$ is generic, i.e. its eigenvalues $\sigma_{i}$ are distinct, then (14) specializes to:

$$
\mathcal{V}_{v_{1}, \ldots, v_{n}}\left(\sigma_{1}, \ldots, \sigma_{n}\right)=\frac{\operatorname{Det}\left\|e^{\mathrm{i} \sigma_{i} v_{j}}\right\|_{i, j=1}^{n}}{\prod_{i<j}\left(\sigma_{i}-\sigma_{j}\right)}
$$

the value of the integral, computed by A. Kirillov, Harish-Chandra, C. Itzykson and J.B. Zuber and many others, in various contexts. The formula 15 for non generic $v$, e.g. for $G=S U(n)$ when some of its eigenvalues coincide, gives zero. More precisely, the symplectic volume of $O_{v}$, the $\sigma \rightarrow 0$ limit of $(15)$ is equal to

$$
v_{v}=\prod_{\alpha>0}\langle\alpha, v\rangle
$$

and vanishes whenever $v$ falls onto a wall of a Weyl chamber. However this zero is merely a reflection of the lower dimensionality of the corresponding orbit $O_{v}=G / G_{v}$. One can take a limit of the normalized equivariant volume $\mathcal{V}_{v} / v_{v}$ for $v$ approaching the wall of the chamber, and arrive at the fixed point formula given by the sum over the coset $W / W_{v}$ with $W_{v}$ the Weyl group of the stabilizer $G_{v}$. For example, for $G=S U(n)$, if the multiplicities of the eigenvalues of $v$ are equal to $\left(n_{1}, \ldots, n_{l}\right)$, with $n_{1}+\ldots+n_{l}=n$, then $G_{v}=S\left(U\left(n_{1}\right) \times \ldots \times U\left(n_{l}\right)\right)$, 
$W_{v}=S\left(n_{1}\right) \times \ldots \times S\left(n_{l}\right)$. The case $(k, n-k)$ corresponds to $O_{v} \approx G r(k, n)$, the Grassmanian of $k$-planes in $\mathbb{C}^{n}$, the $S U(n)$-orbit of a matrix of the form

$$
v \cdot 1_{n}-I I^{\dagger} \quad I: \mathbb{C}^{k} \rightarrow \mathbb{C}^{n}, \quad I^{\dagger} I=\frac{n}{k} v \cdot 1_{k}, \quad v>0
$$

The corresponding integral

$$
\mathcal{V}_{v}\left(\sigma_{1}, \ldots, \sigma_{n}\right)=\sum_{I \subset[n], \# I=k} \prod_{i \in I} \frac{e^{\mathrm{i} v \sigma_{i}}}{\prod_{j \notin I}\left(\sigma_{j}-\sigma_{i}\right)}
$$

The general case is not much more complicated: let $v_{a}$ be the eigenvalue of $v$ of multiplicity $n_{a}, a=1, \ldots, l$, with

$$
\sum_{a=1}^{l} n_{a}=n, \quad \sum_{a=1}^{l} n_{a} v_{a}=0
$$

then

$$
\mathcal{V}_{\vec{v}}\left(\sigma_{1}, \ldots, \sigma_{n}\right)=\sum_{I_{1} \sqcup I_{2} \sqcup \ldots \sqcup I_{l}=[n]} \frac{\prod_{a=1}^{l} \prod_{i \in I_{a}} \operatorname{exp~i} v_{a} \sigma_{i}}{\prod_{1 \leq a<b \leq l} \prod_{i \in I_{a}} \prod_{j \in I_{b}}\left(\sigma_{i}-\sigma_{j}\right)}
$$

with $\# I_{a}=n_{a}$. Note that the denominator has the ghost number

$$
2 \sum_{a<b} n_{a} n_{b}=\operatorname{dim} G / G_{v}
$$

Now let $\Sigma=\Sigma_{g, n}$ be the genus $g$ Riemann surface with $n$ punctures $x_{1}, x_{2}, \ldots, x_{n} \in \Sigma$. Let us assign to the puncture $x_{i}$ a conjugacy class $\left[u_{i}\right] \in G / A d(G)$ represented by $u_{i}=e^{\frac{2 \pi \mathrm{i}}{t}} v_{i} \in T$, and consider the moduli space $\mathcal{M}_{g, n}\left(G ;\left[u_{1}\right], \ldots,\left[u_{n}\right]\right)$ of flat connections with the conjugacy class of holonomy around $x_{i}$ being $\left[u_{i}\right]$. For generic $\left[u_{i}\right]$, and for $\chi\left(\sum_{g, n}\right)=2-2 g-n<0$ this moduli space is a manifold of dimension

$$
\operatorname{dim} \mathcal{M}_{g, n}\left(G ;\left[u_{1}\right], \ldots,\left[u_{n}\right]\right)=2 \operatorname{dim}(G)(g-1)+\sum_{i=1}^{n} \operatorname{dim} O_{v_{i}}=-\chi\left(\sum_{g, n}\right) \operatorname{dim}(G)-\sum_{i=1}^{n} \operatorname{dim}\left(G_{v_{i}}\right)
$$

The theory (1) viewed perturbatively in the parameters $t, u$, can be embedded into the $\mathcal{N}=$ $(2,2)$ supersymmetric Yang-Mills theory. The latter, with the $B$-type topological twist, has additional fields: bosonic $\bar{\sigma}, H$, fermionic $\eta, \chi$, all scalars valued in the adjoint representation of $G$. In the physical theory, $\bar{\sigma}$ is complex conjugate of $\sigma$, in the mathematical applications on often takes $\sigma$ and $\bar{\sigma}$ to be real independent fields.

Define the Wilson point operators, cf. [18]:

$$
\mathcal{O}_{v}(x)=\mathcal{V}_{v}(\sigma(x))
$$

On the one hand, these operators are simply a sophisticated linear combination of the more conventional $\operatorname{Tr}\left(\sigma^{n}\right)$ operators. 
On the other hand, they generate a codimension two defect,

$$
F_{A} \sim \sum_{i=1}^{n} J_{v_{i}} \delta_{x_{i}}^{(2)}
$$

where $J_{v_{i}} \in \mathfrak{g}$ belongs to the (co)adjoint orbit of $v_{i} \in \mathrm{t}^{*}$. Indeed, add the $\mathcal{Q}$-exact term to the action of the $\mathcal{N}=(2,2)$ super-Yang-Mills theory of the form

$$
\mathrm{i} \bar{t} \mathcal{Q} \int_{\Sigma} \operatorname{Tr}(\bar{\sigma} \chi)
$$

and take the limit $\bar{t} \rightarrow \infty$. In this limit the fields $\bar{\sigma}, H \chi, \eta$ decouple, and one is left with the 'physical' Yang-Mills fields $\sigma, A, \psi$ without kinetic term for the fermions $\psi$. Now, integrating out $\sigma$ in the limit of small $u$ one gets precisely (24).

To see that this is something one is really familiar with, let us deform the two dimensional Yang-Mills theory to the gauged G/G Wess-Zumino-Witten model:

$$
\begin{aligned}
& S_{G W Z W}=-\frac{k}{8 \pi} \int_{\Sigma} g^{-1} d_{A} g \wedge \star g^{-1} d_{A} g-\mathrm{i} \Gamma(g, A)= \\
& k S_{W Z W}(g)-\frac{k}{2 \pi} \int_{\Sigma} d^{2} z \operatorname{Tr} A_{z} \partial_{\bar{z}} g g^{-1}+\frac{k}{2 \pi} \int_{\Sigma} d^{2} z \operatorname{Tr} A_{\bar{z}}\left(g^{-1} \partial_{z} g+g^{-1} A_{z} g-A_{z}\right)
\end{aligned}
$$

where $\Gamma(g, A)$ is the gauge invariant extension of the Wess-Zumino term:

$$
\Gamma(g)=\frac{1}{12 \pi} \int_{\partial^{-1} \Sigma} \operatorname{Tr}\left(g^{-1} d g\right)^{3}
$$

Then the Wilson point operators deform to the operators (cf. [17, 49, 91, 12]):

$$
\mathcal{O}_{\lambda}(x)=\operatorname{Tr}_{R_{\lambda}} g(x)
$$

By lifting the gauged WZW theory to the three dimensional Chern-Simons theory on $S^{1} \times \Sigma_{g}$ one can make the whole construction supersymmetric, with the topological supersymmetry reducing to that of the topological Yang-Mills theory in two dimensions [91, 12]:

$$
S_{C S}=\frac{k}{8 \pi} \int_{S^{1} \times \Sigma} \operatorname{Tr}\left(A \wedge d A+\frac{2}{3} A \wedge A \wedge A\right)
$$

The operators (28) correspond to the 'vertical' Wilson loops:

$$
\left\langle\prod_{i} \mathcal{O}_{\lambda_{i}}\left(x_{i}\right)\right\rangle_{G W Z W, \Sigma}=\left\langle\prod_{i} \operatorname{Tr}_{R_{\lambda_{i}}} P \exp \oint_{S_{x_{i}}^{1}} A\right\rangle_{C S, S^{1} \times \Sigma}
$$

Now, the behavior (24) of the curvature is the well-known response of the gauge field on the magnetic source. In Chern-Simons theory the electric sources (Wilson loops) are at the same time magnetic sources, as is well-known. 
The partition function computes for $k>0$ the dimension of the space of conformal blocks,

$$
Z_{g, n}\left(G ;\left[u_{1}\right], \ldots,\left[u_{n}\right]\right)=\sum_{i}(-1)^{i} \operatorname{dim} H^{i}\left(\mathcal{M}_{g, n}\left(G ;\left[u_{1}\right], \ldots,\left[u_{n}\right]\right), \mathcal{L}^{\otimes k}\right),
$$

the celebrated Verlinde formula [119, 17, 49, 125].

The codimension two defects in Chern-Simons theory and in the gauged WZW theory correspond to the vertex operators in the two dimensional conformal field theory, which lives on the boundary of the three dimensional space. It was the dream of finding the analogues of such operators in the higher dimensional analogues of the two dimensional conformal field theories, such as $\mathrm{WZW}_{4}$, that lead to the studies of surface operators in four dimensional supersymmetric gauge theories $[78]^{1}$. The idea, presented in my lecture at the IAS program 'Langlands Program and Physics' in the spring of 2004, was to couple the supersymmetric sigma model with the target space having a G-symmetry, to the four dimensional supersymmetric gauge theory with the gauge group $G$.

\section{Between two and four dimensions}

In this section we pass from gauge theories in two dimensions to gauged sigma models, then generalize sigma models to four dimensional gauge theories.

\subsection{Two dimensional gauged linear sigma models}

A two dimensional $\mathcal{X}$-sigma model on a Riemann surface $C$ is a quantum field theory whose fields are the maps $\phi: C \rightarrow \mathcal{X}$, to some fixed target space $\mathcal{X}$, with the Riemannian metric $\mathrm{G}$. The action, typically, contains a Dirichlet energy term, plus other terms, such as the $B$-field, a tachyon $\mathrm{T}$, and a dilaton $\Phi$ couplings:

$$
S=\int_{C} \operatorname{vol}_{C}(h)\left(\operatorname{tr}\left(h^{-1} \phi^{*} \mathrm{G}\right)+\phi^{*} \mathrm{~T}\right)+\int_{C} \phi^{*} B+\int_{C} \mathcal{R}_{C}(h) \phi^{*} \Phi
$$

Classically, the symmetries of $(\mathcal{X}, \mathrm{G}, B, \mathrm{~T}, \Phi)$ are the global symmetries of the sigma model. Gauging a subgroup of the group of symmetries produces another quantum field theory. Quantum mechanically, some of the classical symmetries may be anomalous, and cannot be consistently gauged.

The gauged WZW model of the previous section is an example with the group manifold as the target space $\mathcal{X}=G$, with the left-right invariant metric $\mathrm{G}=\operatorname{Tr}\left(g^{-1} d g\right)^{2}$, the $B$-field related to the integral generator of $H^{3}(G, \mathbb{Z}), d B=\frac{k}{12 \pi} \operatorname{Tr}\left(g^{-1} d g\right)^{3}$, and the symmetry being the adjoint action of $G$ on itself. The purely left or right $G$-action on itself, albeit a symmetry of the metric $G$, is not a symmetry of the $B$ field.

(Super)symmetry requirements limit the choices of $G, B, \Phi, T$ etc. In particular $\mathcal{N}=(2,2)$ supersymmetry forces the target space to be generalized Kähler. Let us further restrict ourselves with the Kähler case. Let $\left(X^{i}\right)$ denote the local holomorphic coordinates on $\mathcal{X}$, and

\footnotetext{
${ }^{1}$ In order to count the four dimensional generalizations of WZW conformal blocks the supersymmetric localization of five dimensional gauge theory compactified on a circle has been developed [91]. Recently these concepts have resurfaced in 11$]$
} 
$\omega=\frac{1}{2 \mathrm{i}} \mathrm{G}_{i \bar{j}} d X^{i} \wedge d X^{\bar{j}}$ the corresponding symplectic form. Suppose, in addition, that $(\mathcal{X}, \mathrm{G}, \omega)$ has the group $G$ of symmetries (isometries of $G$, symplectomorphisms of $\omega$ ), generated by the vector fields $V_{a}, a=1, \ldots$, dimg, with the moment map $\mu=\left(\mu^{a}\right)$ :

$$
\iota_{V_{a}} \omega=d \mu^{a}, \quad\left\{\mu^{a}, \mu^{b}\right\}=f_{c}^{a b} \mu^{c}=\omega\left(V_{a}, V_{b}\right)
$$

Gauging the G-symmetry in the supersymmetric fashion amounts to coupling the sigma model to the $\mathcal{N}=(2,2)$ supersymmetric gauge theory. The vector multiplet contains, in addition to the gauge field $A=\left(A^{a}\right)$ a complex adjoint-valued scalar $\sigma, \bar{\sigma}$. The theory, in the twisted form, has the nilpotent supercharge, which acts on the basic fields $(A, \psi, \sigma, \bar{\sigma}, \eta, \chi, D)$, $\left(X^{\mu}, \rho^{\mu}, \chi_{i z}, h_{i z}, \chi_{\bar{i} \bar{z}}, h_{\bar{i} \bar{z}}\right)$ of the theory as follows:

\section{adjoint valued}

$$
\begin{aligned}
& \delta A=\psi, \quad \delta \psi=D_{A} \sigma, \quad \delta \sigma=0 \\
& \delta \bar{\sigma}=\eta, \quad \delta \eta=[\bar{\sigma}, \sigma] \\
& \delta \chi=D, \quad \delta D=[\chi, \sigma] \\
& \quad \text { non - linear sigma model } \\
& \delta X^{\mu}=\rho^{\mu}, \quad \delta \rho^{\mu}=V^{\mu}(\sigma)=V_{a}^{\mu} \sigma^{a} \\
& \delta \chi_{i z}=h_{i z}, \quad \delta h_{i z}=-\partial_{i} V^{j}(\sigma) \chi_{j z} \\
& \delta \chi_{\bar{i} \bar{z}}=h_{\bar{i} \bar{z}}, \quad \delta h_{\bar{i} \bar{z}}=-\bar{\partial}_{\bar{i}} V^{\bar{j}}(\sigma) \chi_{\bar{j} \bar{z}}
\end{aligned}
$$

The action of the theory is taken to be:

$$
S=\int_{C} \mathcal{O}_{\omega+\mu}^{(2)}+\delta \int_{C} \Psi
$$

where

$$
\mathcal{O}_{\omega+\mu}^{(2)}=\frac{1}{2} \omega_{\mu \nu} \nabla_{A} X^{\mu} \wedge \nabla_{A} X^{v}+\mathrm{i} \mu\left(F_{A}\right)
$$

is the 2-observable, corresponding to the 0-observable

$$
\mathcal{O}_{\omega+\mu}^{(0)}=\frac{1}{2} \omega_{\mu \nu} \rho^{\mu} \rho^{v}+\mathrm{i} \mu(\sigma)
$$

which are included in the tower $d \mathcal{O}^{(i)}=\delta \mathcal{O}^{(i+1)}$,

$$
\mathcal{O}_{\omega+\mu}^{(1)}=\omega_{\mu \nu} \rho^{\mu} \nabla_{A} X^{v}+\mu(\psi)
$$

In the formulae above we used the notation

$$
\nabla_{A} X^{\mu}=d X^{\mu}+V^{\mu}(A)
$$

One can concisely rewrite (37), 38), 36) as

$$
\mathcal{O}_{\omega+\mu}^{(0)}+\mathcal{O}_{\omega+\mu}^{(1)}+\mathcal{O}_{\omega+\mu}^{(2)}=\frac{1}{2} \omega_{\mu \nu}\left(\rho^{\mu}+\nabla_{A} X^{\mu}\right)\left(\rho^{v}+\nabla_{A} X^{v}\right)+\mu\left(\sigma+\psi+F_{A}\right)
$$

More generally, let

$$
f(X, \rho, \sigma)
$$


be the equivariantly closed differential form on $\mathcal{X}$, then

$$
\mathcal{O}_{f}^{(0)}+\mathcal{O}_{f}^{(1)}+\mathcal{O}_{f}^{(2)}=f\left(X, \rho+\nabla_{A} X, \sigma+\psi+F_{A}\right)
$$

is $\delta$-closed observable in the sigma model.

The bosonic part of the action of the coupled theory has the form:

$$
\begin{aligned}
\int_{C} G_{i \bar{j}}\left(d X^{i}+V_{a}^{i} A^{a}\right) \wedge \star\left(d X^{\bar{j}}+V_{a}^{\bar{j}} A^{a}\right)+\operatorname{Tr} \mu^{2}+\mathrm{G}_{i j} V_{a}^{i} V_{b}^{\bar{j}} \sigma^{a} \bar{\sigma}^{b}+ \\
\quad \operatorname{Tr} D_{A} \sigma \wedge \star D_{A} \bar{\sigma}+\operatorname{Tr}[\sigma, \bar{\sigma}]^{2}+\operatorname{Tr} F_{A} \wedge \star F_{A}
\end{aligned}
$$

The supersymmetric localization locus (it corresponds to the $B$-model twist of the $\sigma, \bar{\sigma}$ multiplet, and the $A$-model twist of the $X^{i}, X^{\bar{j}}$-multiplet) reads:

$$
\begin{aligned}
& \star F_{A}+\mu=0 \\
& \bar{\partial}_{\bar{z}} X^{i}+A_{\bar{z}}^{a} V_{a}^{i}=0 \\
& \sigma^{a} V_{a}^{i}=0 \\
& D_{A} \sigma=0 \\
& {[\sigma, \bar{\sigma}]=0}
\end{aligned}
$$

\subsection{From sigma models to gauge theories}

An interesting class of sigma models in two dimensions have the moduli spaces of solutions to some field equations as their target spaces. Such models arise as limits of higher dimensional field theories. When $X=\mathcal{M}_{g}(G)$ is the moduli space of flat $G$-connections on a genus $g$ Riemann surface $\Sigma_{g}$, the theory $(32)$ is an approximation to the four dimensional gauge theory with gauge group $G$, with the Euclidean spacetime $M^{4}=\Sigma_{g} \times C$, in the limit, where the size of $\Sigma_{g}$ is much smaller then that of $C$. The metric $G$ on $\mathcal{M}_{g}(G)$ is determined by the four dimensional gauge coupling $\mathrm{g}_{\mathrm{ym}}^{2}$, the $B$-field is induced from the four dimensional theta angle $\vartheta$. In the nonsupersymmetric theory, quantum corrections induce the tachyon, which corresponds to a potential on the moduli space, lifting the moduli.

However, if one starts with the supersymmetric gauge theory in four dimensions [13], e.g. $\mathcal{N}=2$ super-Yang-Mills theory, twisted or partially twisted along $\Sigma_{g}$, one gets a twisted or simply supersymmetric sigma model in two dimensions, with $C$ as a worldsheet (of course, for curved $C$ the supersymmetry is broken unless one gives appropriate expectation values to some of the supergravity fields, e.g. the $R$-symmetry gauge field, which amounts to the traditional twisting).

Specifically, the pure $\mathcal{N}=2$ gauge theory in four dimensions with the canonical (DonaldsonWitten) twist gives rise [13] to the $A$ model in two dimensions, whose target space is the moduli space $\mathcal{M}_{g}(G)$ of flat connections. The analysis of [13] breaks down when the moduli space is singular, which means one has to turn on a 't Hooft flux.

However ${ }^{2}$, one can perfectly well study the $A$ model with the moduli space of flat connections on the punctured Riemann surface. These moduli spaces are 'nicer' then those without punctures, and even in genus $g=0$ one can get a smooth space or at least a space with at most orbifold singularities. What kind of four dimensional gauge theory would lead to such a model?

\footnotetext{
${ }^{2}$ Author's remark during C. Vafa lecture at NHETC at Rutgers presenting [13] in 1995
} 


\subsection{The surface defects}

To answer this question we need to learn a little bit about the complex structure of

$$
\mathcal{M}_{g, n}\left(G ;\left[u_{1}\right], \ldots,\left[u_{n}\right]\right) .
$$

Of course, the $A$ model does not care about the choice of the complex structure, but the localization locus of the path integral does. By looking at the localization locus we shall be able to reconstruct the localization locus of the four dimensional theory on $C \times \Sigma_{g}$, which gives the sigma model in the limit of vanishing area of $\Sigma_{g}$. So we pick a complex structure on $\Sigma_{g}$, with local coordinates $z, \bar{z}$. Then the space of all gauge fields $\mathcal{A}_{\Sigma_{g}}$ becomes an infinite dimensional affine space, with the coordinates $A_{\bar{z}}(z, \bar{z})$. The complexification $\mathcal{G}_{\mathbb{C}}$ of the gauge group acts on $\mathcal{A}_{\Sigma_{g}}$ holomorphically:

$$
A_{\bar{z}} \sim g^{-1} A_{\bar{z}} g+g^{-1} \partial_{\bar{z}} g
$$

The quotient $\mathcal{A}_{\Sigma_{g}} / \mathcal{G}_{\mathbb{C}}$ is the moduli stack Bun $G_{\mathbb{C}}\left(\Sigma_{g}\right)$ of holomorphic $G_{\mathbb{C}}$-bundles on $\Sigma_{g}$. However, there is a subset $B u n_{G_{\mathbb{C}}}\left(\Sigma_{g}\right)^{\text {ss }}$ of so-called semi-stable bundles, which is isomorphic to the moduli space $\mathcal{M}_{g}(G)$ of flat $G$-connections. Specifically, it means that $\partial_{\bar{z}}+A_{\bar{z}}$ represents the semi-stable $G_{\mathbb{C}^{-}}$bundle iff one can find $g$ in 45 such that

$$
\left[g^{\dagger}\left(\partial_{z}+A_{z}\right)\left(g^{\dagger}\right)^{-1}, g^{-1}\left(\partial_{\bar{z}}+A_{\bar{z}}\right) g\right]=0
$$

This is the content of Narasimhan-Seshadri's theorem.

Now, with $n$ marked points $x_{1}, \ldots, x_{n} \in \Sigma_{g}$ one can define an additional structure: namely, one choose the so-called parabolic structure at each marked point, i.e. the reduction of the structure group $G_{\mathbb{C}}$ to some parabolic subgroup $P_{i}, i=1, \ldots, n$ (the subgroups may differ at different points). This modifies the definition of stability. The analogue of the NarasimhanSeshadri's theorem in this case identifies the moduli space $B u n_{G_{\mathbb{C}}}\left(\Sigma_{g} ;\left(x_{i}, P_{i}\right)_{i=1}^{n}\right)$ of semi-stable parabolic bundles with that of the moduli space of $G$-flat connections on the punctured surface $\Sigma_{g}$, with the fixed conjugacy classes $\left[u_{i}\right], i=1, \ldots, n$, of holonomy around the punctures.

The parabolic subgroups $P_{i} \subset G_{\mathbb{C}}$ are such that

$$
O_{v_{i}} \approx G_{\mathbb{C}} / P_{i}
$$

as complex manifolds.

Now, on an open dense subset of $B u n_{G_{\mathbb{C}}}\left(\Sigma_{g}\right)$ the choice of the parabolic structure at the marked points is simply a choice of a point in $G_{\mathbb{C}} / P_{i}$ for each $i=1, \ldots, n$, so we get a forgetting map:

$$
f_{n}: \operatorname{Bun}_{G_{\mathbb{C}}}\left(\Sigma_{g} ;\left(x_{i}, P_{i}\right)_{i=1}^{n}\right) \rightarrow \operatorname{Bun}_{G_{\mathbb{C}}}\left(\Sigma_{g}\right)
$$

associating with the parabolic bundle the underlying holomorphic bundle, and $n$ evaluation maps

$$
e_{i}: \operatorname{Bun}_{G_{\mathbb{C}}}\left(\Sigma_{g} ;\left(x_{i}, P_{i}\right)_{i=1}^{n}\right) \longrightarrow G_{\mathbb{C}} / P_{i}
$$

sending the parabolic bundle to the corresponding generalized flag variety. Now, imagine taking the line bundles $\mathcal{L}_{i, \alpha}$ over $G_{\mathbb{C}} / P_{i}$ generating the $K$-theory of the corresponding generalized flag variety, pull back their Chern classes using $e_{i}^{\prime} s$ and push them forward using $f_{n}$. We'd 
get a cohomology class of $B u n_{G_{\mathbb{C}}}\left(\Sigma_{g}\right)$ which one may want to express using the observables of the two-dimensional Yang-Mills theory. The claim is that this is the product of the Wilson point operators we discussed before:

$$
\left(f_{n}\right)_{*} \bigwedge_{i=1}^{n} e_{i}^{*}\left(e^{\sum_{\alpha} v_{i, \alpha} c_{1}\left(\mathcal{L}_{i, \alpha}\right)}\right) \sim \prod_{i=1}^{n} \mathcal{O}_{v_{i}}
$$

Now we introduce $C$ and make everything vary holomorphically over $C$. A collection of holomorphic bundles over $\Sigma_{g}$, which is holomorphically parametrized by $C$ defines a holomorphic bundle over the complex surface $S=C \times \Sigma_{g}$. Moreover, if for each point $w \in C$ the corresponding bundle over $\Sigma_{g}$ is stable, then so is the bundle over $S$. The converse is not necessarily true. Stable bundles over $S$ correspond to the $G$-connections with anti-self-dual curvature:

$$
F_{A}^{+}=\frac{1}{2}\left(F_{A}+\star F_{A}\right)=0
$$

The Hodge star $\star$ on two-forms knows about the conformal structure of $S$. The equations (51) mean that

$$
F_{\bar{z} \bar{w}}=0 \leftrightarrow \partial_{\bar{w}} A_{\bar{z}}=D_{\bar{z}} A_{\bar{w}}
$$

i.e. the holomorphic bundle on $\sum_{g}$ defined by $D_{\bar{z}} \equiv \partial_{\bar{z}}+A_{\bar{z}}$ varies holomorphically in $w$, up to isomorphisms. The equations (51) also imply:

$$
h^{z \bar{z}} F_{z \bar{z}}+h^{w \bar{w}} F_{w \bar{w}}=0
$$

where we assume the hermitian metric on $S$ of the split form:

$$
h_{z \bar{z}} d z d \bar{z}+h_{w \bar{w}} d w d \bar{w}
$$

Taking the limit of small $\sum_{g}$ means taking $h_{z \bar{z}} \rightarrow 0$ while keeping $h_{w \bar{w}}$ finite. In this limit the Eq. (53) means that for almost all $w$ the curvature $F_{z \bar{z}}$ vanishes. However, in a small region of the $w$ space the curvature $F_{z \bar{z}}$ may remain finite. This is the freckled instanton phenomenon [80, 81], well-known in the study of vortex equations. In fact, the four dimensional instanton equations (53) are nothing but the BPS vortex equations written for the gauged linear (actually, affine) sigma model with worldsheet $C$ on $X=\mathcal{A}_{\Sigma_{g}}$ with the infinite-dimensional gauge group $\mathcal{G}$ of two dimensional gauge transformations. The equation $F^{0,2}=0(52)$ is the $A$-model localization locus in the presence of gauge symmetry, while the equation (53) is the $D$-term moment map equation.

Now let us add the parabolic structures. The fact that they also vary holomorphically in $w$ means that we have a collection of holomorphic maps

$$
\varphi_{i}: C \longrightarrow G_{\mathbb{C}} / P_{i}, \quad i=1, \ldots, n
$$

So the four dimensional lift of the $A$ model on $\mathcal{M}_{g, n}\left(G ;\left[u_{1}\right], \ldots,\left[u_{n}\right]\right)$ looks like a four dimensional $\mathcal{N}=2$ super-Yang-Mills theory coupled to the two dimensional $A$ model on

$$
X=\searrow_{i=1}^{n} G_{\mathbb{C}} / P_{i}
$$


We can view the combined theory again as the gauged linear sigma model with

$$
X=\mathcal{A}_{\Sigma_{g}} \times \searrow_{i=1}^{n} G_{\mathbb{C}} / P_{i}
$$

where the gauged group is the infinite dimensional group $\mathcal{G}$ of gauge transformations on $\Sigma_{g}$. Then the $A$-model localization locus would amount to:

$$
\begin{aligned}
& F^{0,2}=0 \quad \text { outside } \quad C \times\left\{x_{1}, \ldots x_{n}\right\} \\
& \partial_{\bar{w}} \xi_{i}=0, \quad \xi_{i}=\text { holomorphic coordinates on } \quad G_{\mathbb{C}} / P_{i}
\end{aligned}
$$

and the $D$-term equation

$$
h^{z \bar{z}}\left(F_{z \bar{z}}+\sum_{i=1}^{n} J_{v_{i}} \delta^{(2)}\left(x_{i}\right)\right)+h^{w \bar{w}} F_{w \bar{w}}=0
$$

We have arrived at the surface operators in gauge theory. They were introduced in [73, 74]. See [62, 63] for the recent discussion in the context of supersymmetric gauge theories.

\section{Supersymmetric count and partition functions}

In this section we study two dimensional gauged linear sigma model with $\mathcal{N}=(4,4)$ supersymmetry, which is softly broken to $\mathcal{N}=(2,2)$ by the twisted mass corresponding to the specific $U(1)$-symmetry.

\subsection{Twisted Witten index and generalized Hitchin equations}

We want to compute the partition function of this theory in the $\Omega$-background with the parameter $\varepsilon$. In other words, we deform the theory by first lifting it to three dimensions and then compactifying on an $\mathbb{R}^{2}$ (or $D^{2}$ ) -bundle over $S^{1}$ of small circumference $r$ with the rotation of the fiber by the angle $\varepsilon r$. Then we take the $r \rightarrow 0$ limit to get back the two dimensional theory. In addition to the $\mathbb{R}^{2}$-rotation, we twist the boundary conditions by the global gauge and flavor

$$
\begin{aligned}
& \mathrm{g}_{\text {gauge }}=e^{\mathrm{i} r \sigma}, \quad \sigma \in \mathrm{t} \subset \mathfrak{g} \\
& \mathrm{g}_{\text {flavor }}=e^{\mathrm{i} r m}, \quad m \in \operatorname{Lie}\left(G_{\text {flavor }}\right)
\end{aligned}
$$

transformations. We compute

$$
\mathcal{Z}_{3 d}=\operatorname{Tr}\left((-1)^{F} e^{-r \hat{H}} g_{\text {gauge }} \mathrm{g}_{\text {flavor }} e^{\mathrm{i} \varepsilon r \hat{J}_{\text {rot }}}\right)
$$

Here $\hat{J}_{\text {rot }}$ is a combination of the generator of the $\mathbb{R}^{2}$ rotation and the $R$-symmetry which makes the twisted boundary conditions compatible with some supersymmetry. The specific twist we employ makes the bosonic fields of vector multiplet into $\left(A_{z} d z+A_{\bar{z}} d \bar{z}, \Phi_{z} d z+\Phi_{\bar{z}} d \bar{z}, \sigma, \bar{\sigma}\right)$, while the bosonic fields of the hypermultplet $(Q, \tilde{Q})$ and their complex conjugates $\left(Q^{*}, \tilde{Q}^{*}\right)$ remain scalars. Here $A_{\bar{z}}$ is the $(0,1)$-component of a $G$-gauge field, with the compact Lie group $G$, ie. a connection on a principal $G$-bundle $\mathcal{P} \rightarrow \Sigma, \Phi_{z} d z$ is the $(1,0)$-form valued in the adjoint vector bundle,

$$
\Phi_{z} d z \in \Gamma\left(\Omega_{\Sigma}^{1,0} \otimes \mathfrak{g}_{\mathcal{P}}\right),
$$


$Q$ is a section of a vector bundle $R_{\mathcal{P}}$, associated with the (complex) representation $R$ of $G$, and $\tilde{Q}$ is a section of a vector bundle $R_{\mathcal{P}}^{*}$, associated with the dual representation. Let

$$
\mu_{\mathbb{R}} \oplus \mu_{\mathbb{C}}: R \oplus R^{*} \longrightarrow \mathfrak{g}^{*} \otimes(\mathbb{R} \oplus \mathbb{C})
$$

be the hyperkähler moment map

$$
\begin{aligned}
& \mu_{\mathbb{R}}^{\mathbf{a}}=\left(T_{R}^{\mathbf{a}}\right)_{i}^{j}\left(Q^{i} Q_{j}^{*}-\tilde{Q}_{j} \tilde{Q}^{*, i}\right)-\zeta_{\mathbb{R}}^{\mathbf{a}} \\
& \mu_{\mathbb{C}}^{\mathbf{a}}=\left(T_{R}^{\mathbf{a}}\right)_{i}^{j} Q^{i} \tilde{Q}_{j}-\zeta_{\mathbb{C}}^{\mathbf{a}}
\end{aligned}
$$

where $\zeta_{\mathbb{R}}, \zeta_{\mathbb{C}}$ are the real and the complex Fayet-Iliopoulos terms, valued in the centralizer of $\mathfrak{g}$.

The partition function localizes onto the integral over the moduli space $\mathcal{M}$ of solutions to the generalized vortex equations (recall that $Q^{\dagger}=Q^{*}, \tilde{Q}^{\dagger}=\tilde{Q}^{*}, \Phi_{z}^{\dagger}=\Phi_{\bar{z}}$ ):

$$
\begin{aligned}
& D_{\bar{z}} Q \equiv \partial_{\bar{z}} Q+T_{R}\left(A_{\bar{z}}\right) \cdot Q=\left(T_{R^{*}}\left(\Phi_{z}\right) \cdot \tilde{Q}\right)^{\dagger} \\
& D_{\bar{z}} \tilde{Q}=\left(T_{R}\left(\Phi_{z}\right) \cdot Q\right)^{\dagger} \\
& D_{\bar{z}} \Phi_{z}+\mu_{\mathbb{C}}^{\dagger} \operatorname{Vol}_{\Sigma}=0 \\
& F_{z \bar{z}}+\left[\Phi_{z}, \Phi_{\bar{z}}\right]+\mu_{\mathbb{R}} \operatorname{vol}_{\Sigma}=0
\end{aligned}
$$

modulo gauge transformations

$$
(A, \Phi, Q, \tilde{Q}) \mapsto\left(g^{-1} A g+g^{-1} d g, g^{-1} \Phi g, T_{R}\left(g^{-1}\right) \cdot Q, T_{R^{*}}\left(g^{-1}\right) \cdot \tilde{Q}\right) .
$$

More specifically, we wish to study the equivariant index of Dirac operator on $\mathcal{M}^{\text {framed }}$, possibly twisted with a vector bundle. Here the superscript "framed" means that the gauge symmetry group is reduced, by requiring $g$ in (66) to tend to 1 at infinity. Instead of solving the Eqs. (65) and studying the Dirac operator on the space of solutions, we modify the Dirac operator on the ambient space $M$, by coupling it to the exterior powers $\Lambda^{i} E$ of the vector bundle $E$ where the Eqs. (65) take values: $\not D \longrightarrow \not D \otimes 1_{\Lambda^{i} E}+1 \otimes \Lambda^{i}$ s. Here we represent 65) collectively by $s=0$, with $s: M \rightarrow E$. The indices of these operators are then summed over all values of $i$ with the sign $(-1)^{i}$. The result is the index of Dirac operator restricted onto the zero locus of $s$, since the Chern character of $\sum_{i}(-1)^{i} \Lambda^{i} E$ is the Poincare dual of the $s=0$ locus. One can make a more detailed argument using the supersymmetric quantum mechanics representation.

\subsection{Quiver gauge theories}

In this paper the gauge group and the matter representation corresponds to a quiver $\gamma$. The gauge group

$$
G_{\text {gauge }}=\chi_{i \in \mathrm{V}_{\gamma}} U\left(\mathbf{N}_{i}\right)
$$

is the product of unitary groups corresponding to the vertices of the quiver, the representation:

$$
R=\bigoplus_{i \in \mathrm{V}_{\gamma}} \operatorname{Hom}\left(\mathbf{M}_{i}, \mathbf{N}_{i}\right) \oplus \bigoplus_{e \in \mathrm{E}_{\gamma}} \operatorname{Hom}\left(\mathbf{N}_{s(e)}, \mathbf{N}_{t(e)}\right)
$$


with $\mathbf{M}_{i}, i \in \mathrm{V}_{\gamma}$, the spaces of multiplicities of the fundamental hypermultiplets.

The computation of (61) can be done simply by enumerating all holomorphic functions of $A_{\bar{z}}(z, \bar{z}), \Phi_{z}(z, \bar{z}), Q(z, \bar{z}), \tilde{Q}(z, \bar{z})$ modulo linearized gauge symmetries and equations (65), treating $z$, $\bar{z}$-dependence formally. This is a free field character, given by the plethystic exponent

$$
\mathcal{Z}_{3 d}=\exp -\sum_{l=1}^{\infty} \frac{1}{l} F\left(q^{l}, \mathrm{~g}_{\text {gauge }}^{l}, \mathrm{~g}_{\text {flavor }}^{l}\right)
$$

where 3

$$
F\left(q, \text { g gauge }_{\text {gflavor }}\right)=\frac{\chi_{\text {fields }}-\chi_{\text {symmetries }}-\chi_{\text {equations }}}{(1-q)\left(1-q^{-1}\right)}
$$

with

$$
\begin{aligned}
& \chi_{\text {fields }}=\sum_{i \in \mathrm{V}_{\gamma}}\left\{\left(q^{-1}+q t^{2}\right) N_{i} N_{i}^{*}+t^{-1}\left(M_{i} N_{i}^{*}+N_{i} M_{i}^{*}\right)\right\}+\sum_{e \in \mathrm{E}_{\gamma}} t^{-1}\left(N_{s(e)} N_{t(e)}^{*}+N_{t(e)} N_{s(e)}^{*}\right) \\
& \chi_{\text {symmetries }}=\sum_{i \in \mathrm{V}_{\gamma}} N_{i} N_{i}^{*} \\
& \chi_{\text {equations }}=\sum_{i \in \mathrm{V}_{\gamma}}\left\{t^{2} N_{i} N_{i}^{*}+q^{-1} t^{-1}\left(M_{i} N_{i}^{*}+N_{i} M_{i}^{*}\right)\right\}+\sum_{e \in \mathrm{E}_{\gamma}} q^{-1} t^{-1}\left(N_{s(e)} N_{t(e)}^{*}+N_{t(e)} N_{s(e)}^{*}\right)
\end{aligned}
$$

Here $q=e^{\mathrm{i} \varepsilon r}$ is the rotation eigenvalue, $t=e^{\mathrm{i} r u}$ is the soft $\mathcal{N}=4 \longrightarrow \mathcal{N}=2$ supersymmetry breaking phase corresponding to the $U(1)$ symmetry under which both $Q$ and $\tilde{Q}$ have charges +1 , while $\Phi_{z}$ has charge -2 ,

$$
\begin{array}{ll}
N_{i}=\operatorname{Tr}_{\mathbf{N}_{i}} \mathbf{g}_{\text {gauge }}, & N_{i}^{*}=\operatorname{Tr}_{\mathbf{N}_{i}} \mathbf{g}_{\text {gauge }}^{\dagger} \\
M_{i}=\operatorname{Tr}_{\mathbf{M}_{i}} \mathbf{g}_{\text {flavor }}, & M_{i}^{*}=\operatorname{Tr}_{\mathbf{M}_{i}} \mathbf{g}_{\text {flavor }}^{+}
\end{array}
$$

Recall the formula for local contribution to the equivariant index of Dirac operator:

$$
\prod_{\alpha} \frac{1}{2 \sinh \left(\frac{\xi_{\alpha}}{2}\right)}=e^{-\frac{1}{2} \sum_{\alpha} \xi_{\alpha}} \exp \left(-\sum_{l=1}^{\infty} \frac{1}{l} \sum_{\alpha} e^{-l \xi_{\alpha}}\right)
$$

Since changing the sign of $\xi_{\alpha}$ simply changes the overall sign of the contribution 73 we can, modulo the overall sign ambiguity, rewrite (61) in a compact form (by replacing some of the characters by their duals):

$$
F \sim \frac{1-q t^{2}}{1-q}\left\{\sum_{i \in \mathrm{V}_{\gamma}}\left(t^{-1} M_{i}-N_{i}\right) N_{i}^{*}+\sum_{e \in \mathrm{E}_{\gamma}} t^{-1} N_{s(e)} N_{t(e)}^{*}\right\}
$$

In what follows we shall use the notation (cf. [98])

$$
\epsilon\left[F^{(\tau)}\right]=\left.\exp \frac{d}{d s}\right|_{s=0} \frac{1}{\Gamma(s)} \int_{0}^{\infty} \frac{d \tau}{\tau} \tau^{s} F^{(\tau)}
$$

\footnotetext{
${ }^{3}$ The denominator $(1-q)\left(1-q^{-1}\right)$ comes from the $z, \bar{z}$-dependence of the fields $A_{\bar{z}}(z, \bar{z})$, etc. e.g. the mode $z^{i} \bar{z}^{j} d \bar{z}$ contributes $q^{i}\left(q^{-1}\right)^{j} q^{-1}$
} 
In the limit $r \rightarrow 0$, the plethystic sum in 61 over $l$ becomes a proper time integral:

$$
\mathcal{Z}_{2 d}^{\text {pert }}=\epsilon\left[\frac{1-e^{\tau(\varepsilon+2 u)}}{1-e^{\tau \varepsilon}}\left\{\sum_{i \in \mathrm{V}_{\gamma}}\left(e^{-\tau u} M_{i}^{(\tau)}-N_{i}^{(\tau)}\right) N_{i}^{(-\tau)}+\sum_{e \in \mathrm{E}_{\gamma}} e^{-\tau u} N_{s(e)}^{(\tau)} N_{t(e)}^{(-\tau)}\right\}\right]
$$

with

$$
N_{i}^{(\tau)}=\sum_{\alpha=1}^{n_{i}} e^{\tau a_{i, \alpha}}, \quad M_{i}^{(\tau)}=\sum_{f=1}^{\mathrm{rk} \mathbf{M}_{i}} e^{\tau m_{i, f}}
$$

(with $n_{i}=\mathrm{rkN}_{i}$ ) which becomes a product of $\Gamma$-functions (cf. 98, In (76) we put the superscript pert to stress the fact that this is only a perturbative, i.e. one-loop exact, contribution to the partition function.

The full partition function is not much more complicated. Namely, we sum over the flux sectors, where the gauge field for each of the $U(1)$ subgroups of each of the $U\left(\mathbf{N}_{i}\right)$ groups may have a non-vanishing flux $F_{i, \alpha}$, with

$$
\int_{\mathbb{R}^{2}} F_{i, \alpha}=2 \pi \mathrm{i} d_{i, \alpha}, \quad d_{i, \alpha} \in \mathbb{Z}
$$

The instanton contribution to the partition function is computed, e.g. with the help of the family index theorem, as:

$$
\mathcal{Z}_{2 d}=\sum_{\left(\mathbf{d}_{i}\right)_{i \in \mathrm{V}_{\gamma}}}\left(\prod_{i \in \mathrm{V}_{\gamma}} \mathfrak{q}_{i}^{\left|\mathbf{d}_{i}\right|}\right) \cdot \epsilon\left[\frac{1-e^{\tau(\varepsilon+2 u)}}{1-e^{\tau \varepsilon}}\left\{\sum_{i \in \mathrm{V}_{\gamma}}\left(e^{-\tau u} M_{i}^{(\tau)}-\Sigma_{i}^{(\tau)}\right) \Sigma_{i}^{(-\tau)}+\sum_{e \in \mathrm{E}_{\gamma}} e^{-\tau u} \sum_{s(e)}^{(\tau)} \Sigma_{t(e)}^{(-\tau)}\right\}\right]
$$

with $\mathbf{d}_{i}=\left(d_{i, \alpha}\right)_{\alpha \in\left[n_{i}\right]}, \mathfrak{q}_{i}$ being the exponentiated Kähler moduli,

$$
\left|\mathbf{d}_{i}\right|=\sum_{\alpha} d_{i, \alpha}
$$

and

$$
\sum_{i}^{(\tau)}=\sum_{\alpha} e^{\tau \sigma_{i, \alpha}}
$$

with

$$
\sigma_{i, \alpha}=a_{i, \alpha}+\varepsilon d_{i, \alpha} .
$$

The range of the fluxes $d_{i, \alpha}$ actually depends on the stability data. In our examples below these will be simply $d_{i, \alpha} \geq 0$.

By playing with the $u$ and $m_{i, f}$ parameters one can get the more general $\mathcal{N}=(2,2)$ quiver gauge theory partition function. In this case the quiver edges (now one allows multiple edges connecting two vertices, possibly with the opposite orientations) correspond to the bifundamental chiral multiplets, while the nodes come with two types of multiplicities, $\mathbf{M}_{i, \pm}$, for the fundamental, and the anti-fundamental chiral multiplets, respectively. The partition 
function is equal to, then:

$$
\begin{aligned}
& \mathcal{Z}_{2 d}^{\mathcal{N}=2}= \\
& \sum_{\left(\mathbf{d}_{i}\right)_{i \in \mathrm{V}_{\gamma}}}\left(\prod_{i \in \mathrm{V}_{\gamma}} \mathfrak{q}_{i}^{\left|\mathbf{d}_{i}\right|}\right) \cdot \epsilon\left[\frac{1}{1-e^{\tau \varepsilon}}\left\{\sum_{i \in \mathrm{V}_{\gamma}}\left(M_{i,+}^{(\tau)} \Sigma_{i}^{(-\tau)}+M_{i,-}^{(-\tau)} \Sigma_{i}^{(\tau)}-\Sigma_{i}^{(\tau)} \Sigma_{i}^{(-\tau)}\right)+\sum_{e \in \mathrm{E}_{\gamma}} e^{\tau m_{e}} \sum_{s(e)}^{(\tau)} \Sigma_{t(e)}^{(-\tau)}\right\}\right] \\
& \cdot \epsilon\left[\frac{1}{1-e^{\tau \varepsilon}}\left\{\sum_{i \in \mathrm{V}_{\gamma}} e^{\tau \varepsilon}\left(\tilde{M}_{i,+}^{(\tau)} \sum_{i}^{(-\tau)}+\tilde{M}_{i,-}^{(-\tau)} \sum_{i}^{(\tau)}\right)+\sum_{\tilde{e} \in \tilde{E}_{\gamma}^{\tilde{r}}} e^{\tau\left(\tilde{m}_{\tilde{e}}+\varepsilon\right)} \sum_{s(\tilde{e})}^{(\tau)} \sum_{t(\tilde{e})}^{(-\tau)}\right\}\right]
\end{aligned}
$$

Here we separated the contributions of the chiral multiplets whose bosonic components remain scalars on $C$ and the chiral multiplets (whose multiplicity spaces and masses have tildes) which become 1-forms. In order to preserve the topological supersymmetry the superpotential $W$ (which comes from the cycles in the quiver) is a $(1,0)$-form on the worldsheet (not to be confused with the twisted superpotential $\tilde{W})$. For example, in the $\mathcal{N}=(4,4)$ case it has the form:

$$
W=\mu_{\mathbb{C}}\left(\Phi_{z}\right) d z
$$

The possible twists (such as the one making the adjoint chiral $\Phi$ a one-form $\Phi_{z}$ ) are encoded in the $\varepsilon$-dependence of the (anti-)fundamental masses in $M_{i, \pm}$ and the bi-fundamental masses $m_{e}$

- An important remark is in order. It would appear that the partition functions 79 , 83 depend on both the Coulomb parameters $a_{i, \alpha}$ and the masses $m_{e}, m_{i, f}$. However, in two dimensions one does not expect to be able to freely fix the scalars in the vector multiplet. Instead, they are dynamically fixed by the choice of the (generically massive) vacuum the theory falls in the infrared. In practice this means that one actually should set $a_{i, \alpha}=a_{i, \alpha}(m)$ to be equal to the classical values determined by the twisted masses. These are discrete choices, which correspond to the choice of the equivariant cohomology class of the effective target space.

\subsection{Twisted superpotential and supersymmetric ground states}

The vacua of the $\mathcal{N}=(2,2)$ theory can be then determined by finding a saddle point in the sum over the fluxes in the limit $\varepsilon \rightarrow 0$, as is done in four dimensions [108, 102]:

$$
\mathcal{Z}_{2 d}^{\mathcal{N}=2} \sim \exp \frac{1}{\varepsilon} \tilde{\mathcal{W}}(\sigma)+\ldots
$$

where $\sigma_{i, \alpha}$ are found from the Bethe-like equations

$$
\frac{P_{i,+}\left(\sigma_{i, \alpha}\right)}{P_{i,-}\left(\sigma_{i, \alpha}\right)} \frac{\tilde{P}_{i,+}\left(\sigma_{i, \alpha}\right)}{\tilde{P}_{i,-}\left(\sigma_{i, \alpha}\right)} \frac{\prod_{e \in t^{-1}(i)} Q_{s(e)}\left(\sigma_{i, \alpha}-m_{e}\right)}{\prod_{e \in s^{-1}(i)} Q_{t(e)}\left(\sigma_{i, \alpha}+m_{e}\right)} \frac{\prod_{\tilde{e} \in t^{-1}(i)} Q_{s(\tilde{e})}\left(\sigma_{i, \alpha}-\tilde{m}_{\tilde{e}}\right)}{\prod_{\tilde{e} \in s^{-1}(i)} Q_{t(\tilde{e})}\left(\sigma_{i, \alpha}+\tilde{m}_{\tilde{e}}\right)}=\mathfrak{q}_{i}
$$

where $e \in \mathrm{E}_{\gamma}, \tilde{e} \in \tilde{\mathrm{E}_{\gamma}}$, for all $\alpha=1, \ldots, n_{i}$, and

$$
P_{i, \pm}(x)=\prod_{f=1}^{m_{i, \pm}}\left(x-m_{ \pm, f}\right), \quad Q_{i}(x)=\prod_{\alpha=1}^{n_{i}}\left(x-\sigma_{i, \alpha}\right), \tilde{P}_{i, \pm}(x)=\prod_{f=1}^{\tilde{m}_{i, \pm}}\left(x-\tilde{m}_{ \pm, f}\right) .
$$


The classical values $\left(a_{i, \alpha}(m)\right)$ are the poles of the meromorphic form:

$$
\bigwedge_{i, \alpha} d x_{i, \alpha} \prod_{i \in \mathrm{V}_{\gamma}} \prod_{\alpha \neq \beta}\left(x_{i, \alpha}-x_{i, \beta}\right) \prod_{\alpha=1}^{n_{i}} \frac{\tilde{P}_{i,+}\left(x_{i, \alpha}\right) \tilde{P}_{i,-}\left(x_{i, \alpha}\right)}{P_{i,+}\left(x_{i, \alpha}\right) P_{i,-}\left(x_{i, \alpha}\right)} \frac{\prod_{\tilde{e} \in \tilde{\mathrm{E}}_{\gamma}} \prod_{\alpha \in\left[n_{s(\tilde{e})}\right]} \prod_{\beta \in\left[n_{t(\tilde{e})}\right.}\left(x_{s(\tilde{e}), \alpha}-x_{t(\tilde{e}), \beta}+\tilde{m}_{\tilde{e}}\right)}{\prod_{e \in \mathrm{E}_{\gamma}} \prod_{\alpha \in\left[n_{s(e)}\right]} \prod_{\beta \in\left[n_{t(e)]}\right.}\left(x_{s(e), \alpha}-x_{t(e), \beta}+m_{e}\right)}
$$

Let us recall the effective twisted superpotential for the softly broken $\mathcal{N}=(4,4)$ theories

$$
\begin{aligned}
\tilde{\mathcal{W}}(\sigma ; \mu ; u) & =\sum_{i \in \mathrm{V}_{\gamma}} \sum_{\alpha \in\left[n_{i}\right]}\left(\log \left(\mathfrak{q}_{i}\right) \sigma_{i, \alpha}\right. \\
& +\sum_{\beta \in\left[n_{i}\right]} \omega\left(-2 u+\sigma_{i, \alpha}-\sigma_{i, \beta}\right)+ \\
& \left.+\sum_{\mathrm{f} \in\left[m_{i}\right]}\left(\omega\left(u+\sigma_{i, \alpha}-m_{i, \mathrm{f}}\right)+\omega\left(u-\sigma_{i, \alpha}+m_{i, \mathrm{f}}\right)\right)\right) \\
& +\sum_{e \in \mathrm{E}_{\gamma}} \sum_{\alpha \in\left[n_{t(e)}\right]} \sum_{\beta \in\left[n_{s(e)}\right]}\left(\omega\left(u+m_{e}+\sigma_{t(e), \alpha}-\sigma_{s(e), \beta}\right)+\omega\left(u-m_{e}+\sigma_{s(e), \beta}-\sigma_{t(e), \alpha}\right)\right)
\end{aligned}
$$

where

$$
\omega(z)=z(\log (z)-1), \quad \exp \omega^{\prime}(z)=z
$$

The equations 86 describe the generalized critical points of $\tilde{\mathcal{W}}$ :

$$
\exp \frac{\partial \tilde{\mathcal{W}}}{\partial \sigma_{i, \alpha}}=1
$$

- Note that our partition functions are the sums over discrete sets. Since these partition functions are obtained by the equivariant integration over the moduli spaces which can be given a finite-dimensional Kähler quotients, they can also be given a contour integral expression a la [83. The asymptotics (85) then becomes the analogue of the critical level limit of the integral representation (free field) of the solutions to KZ equations [29, 48, 17, 9, 10, 118, 39. The connection to Bethe ansatz equations is at first surprising and forms the basis of the Bethe/gauge-correspondence [106, 107, 108. Its mathematical foundations are laid out in 82.

\subsection{Sigma models on grassmanians and vector bundles over them}

The following examples are important:

- The Grassmanian $\operatorname{Gr}(n, m)$ sigma model: it is the $A_{1}$ type theory, with the quiver consisting of one node, with $\mathbf{M}_{+}=\mathbb{C}^{m}, \mathbf{N}=\mathbb{C}^{n}, \mathbf{M}_{-}=0$,

$$
M_{+}^{(\tau)}=\sum_{f=1}^{m} e^{\tau m_{f}} .
$$

A simple computation shows

$$
\frac{\mathcal{Z}_{2 d}}{\mathcal{Z}_{2 d}^{\text {pert }}}=\sum_{d_{1}, \ldots, d_{n} \geq 0} \mathfrak{q}^{d_{1}+d_{2}+\ldots+d_{n}} J_{\vec{d}}^{G r}
$$


where

$$
\begin{aligned}
J_{\vec{d}}^{G r}=\epsilon\left[\frac{\left(M_{+}^{(\tau)}-\Sigma^{(\tau)}\right) \Sigma^{(-\tau)}-\left(M_{+}^{(\tau)}-N^{(\tau)}\right) N^{(-\tau)}}{1-e^{\tau \varepsilon}}\right]= \\
\prod_{1 \leq i<j \leq n} \frac{a_{i}-a_{j}+\varepsilon\left(d_{i}-d_{j}\right)}{a_{i}-a_{j}} \prod_{f=1}^{m} \prod_{i=1}^{n} \prod_{l=1}^{d_{i}} \frac{1}{a_{i}-m_{f}+\varepsilon l}
\end{aligned}
$$

which agrees with [14] (with $\hbar=-\varepsilon$ ) provided we view the partition function (93) as valued in the $U(m)$-equivariant cohomology of $\operatorname{Gr}(n, m)$, which is the ring of $S(n)$-invariant polynomials in $a_{1}, \ldots, a_{n}$ subject to the relation

$$
A(x) B(x)=P(x)=\prod_{f=1}^{m}\left(x-m_{f}\right)
$$

where $A(x)=\prod_{i=1}^{n}\left(x-a_{i}\right)$ and the degree $m-n$ polynomial $B(x)$ is determined from 95 (see also [66] for the period interpretation of (94) in the context of mirror symmetry). As the $\mathbb{C}\left[m_{f}\right]^{S(m)}$-module the ring has the rank $\left(\begin{array}{c}m \\ n\end{array}\right)$.

- The next example is the gauged linear sigma model corresponding to the vector bundle $F$ over $G r(n, m)$, with the fiber $\operatorname{Hom}\left(\mathcal{E}, \mathbf{M}_{-}\right)$being $k$ copies of the dual to the tautological rank $n$ bundle $\mathcal{E}^{\vee}$ over $\operatorname{Gr}(n, m)$. In our conventions this model corresponds to $\mathbf{M}_{+}=\mathbb{C}^{m}, \mathbf{M}_{-}=\mathbb{C}^{k}$. Let

$$
\begin{aligned}
& M_{+}^{(\tau)}=\sum_{f=1}^{m} e^{\tau m_{+, f}}, \quad M_{-}^{(\tau)}=\sum_{f=1}^{k} e^{\tau m_{-, f},} \\
& P_{+}(x)=\prod_{f=1}^{m}\left(x-m_{+, f}\right), \quad P_{-}(x)=\prod_{f=1}^{k}\left(x-m_{-, f}\right)
\end{aligned}
$$

The instanton partition function evaluates to (up to the overall sign and a possible redefinition $\mathfrak{q} \rightarrow-\mathfrak{q})$ :

$$
\frac{\mathcal{Z}_{2 d}}{\mathcal{Z}_{2 d}^{\text {pert }}}=\sum_{d_{1}, \ldots, d_{n} \geq 0} \mathfrak{q}^{d_{1}+d_{2}+\ldots+d_{n}} J_{\vec{d}}^{F}
$$

with

$$
J_{\vec{d}}^{F}=\prod_{1 \leq i<j \leq n} \frac{a_{i}-a_{j}+\varepsilon\left(d_{i}-d_{j}\right)}{a_{i}-a_{j}} \prod_{i=1}^{n} \prod_{l=1}^{d_{i}} \frac{P_{-}\left(a_{i}+\varepsilon(l-1)\right)}{P_{+}\left(a_{i}+\varepsilon l\right)}
$$

where $a_{1} \neq a_{2} \neq \ldots \neq a_{n}$ are to be chosen from the roots of $P_{+}$.

- The general Nakajima varieties, which are the hyperkähler quotients of the space of quiver representations will be discussed in this framework in our future work. We plan to connect our approach to the beautiful results of [20], [1] and especially [82].

\section{Noncommutative description}

In this section we explain how one can derive our formula (79) (as well as the formulae for the four dimensional instanton partition functions [94, 96, 97]) using noncommutative gauge 
theory, which is, in some sense, a minimal model of the open string field theory [116]. The gauged linear sigma model for an affine ADE type quiver can be realized as the low energy limit of the theory of open strings ending on a collection of $D$-branes wrapping various cycles on an ADE singularity, and two noncompact directions transverse to it. The equivariant parameters $u$ and $\varepsilon$ are realized geometrically. The full ten dimensional geometry is $S_{A D E} \times$ $B_{u} \times D_{\varepsilon} \times T^{2}$ where $B_{u} \approx \mathbb{C}, D_{\varepsilon} \approx \mathbb{C}$ are fibered holomorphically over $T^{2}=E$, with complex parameters $u$ and $\varepsilon$ being simply the points on the Jacobian $\operatorname{Jac}(E)$. The factor $S_{A D E}$ is the complex surface which is a resolution of singularities of $L \otimes \mathbb{C}^{2} / \Gamma_{\gamma}$, with $L^{\otimes-2} \approx B_{u} \otimes D_{\varepsilon}$ as line bundles over $E$, where $\Gamma_{\gamma} \subset S U(2)$ is a finite subgroup, whose representations are encoded by the quiver.

By turning an appropriate $B$-field on $S_{A D E} \times B_{u} \times D_{\varepsilon}$ and taking the Seiberg-Witten limit [116] one arrives at the theory which can be roughly described as the dimensional reduction of the $\mathcal{N}=1, d=10$ super-Yang-Mills theory down to two dimensions (with the worldsheet being $T^{2}$ ), with a peculiar gauge group: a group of unitary transformations of a Hilbert space $\mathcal{H}$. This space is described as follows.

Let $\mathcal{H}_{2}$ be the Fock space of states of two harmonic oscillators:

$$
\mathcal{H}_{2}=\mathbb{C}\left[\mathbf{a}_{1}^{\dagger}, \mathbf{a}_{2}^{\dagger}\right]|\mathrm{vac}\rangle
$$

with the operators $\mathbf{a}_{1}, \mathbf{a}_{2}, \mathbf{a}_{1}^{\dagger}, \mathbf{a}_{2}^{\dagger}$ obeying the Heisenberg algebra $\left[\mathbf{a}_{i}, \mathbf{a}_{j}^{\dagger}\right]=\delta_{i j}, i, j=1,2,\left[\mathbf{a}_{1}, \mathbf{a}_{2}\right]=$ 0 .

Let $\underline{4}, \underline{\mathbf{6}}$ be the sets defined in [98]. For $a \in \underline{\mathbf{4}}, A \in \underline{\mathbf{6}}$, such that $a \in A$ define $i(a, A)$ to be 1 if $A=a b$ with $a<b$ and 2 if $a>b$. Fix six Hermitian vector spaces $N_{A}, A \in \underline{\mathbf{6}}$.

Let $\mathcal{H} \approx \mathbf{N} \otimes \mathcal{H}_{2}$, with $\mathbf{N}=\bigoplus_{A} N_{A}, A \in \underline{\mathbf{6}}$. Define

$$
A_{a}=\frac{1}{\sqrt{2}} \bigoplus_{A \in \underline{\underline{6}}, a \in A} 1_{N_{A}} \otimes \mathbf{a}_{i(a, A)}
$$

In other words, we have distributed two creation operators between six copies of the Fock space tensored with some finite-dimensional vector space. Then

$$
\left[A_{a}, A_{b}\right]=0, \quad\left[A_{a}, A_{b}^{\dagger}\right]=\frac{1}{2} \delta_{a b} \bigoplus_{A \in \underline{\mathbf{6}}, A \ni a} 1_{N_{A}}
$$

Now let us look for the quadruples of operators $Z_{a}, a \in \underline{4}$, obeying a weaker version of (101):

$$
\begin{gathered}
{\left[Z_{a}, Z_{b}\right]+\varepsilon_{a b c d}\left[Z_{c}, Z_{d}\right]^{\dagger}=0,} \\
\sum_{a \in \underline{4}}\left[Z_{a}, Z_{a}^{\dagger}\right]=1_{\mathcal{H}}
\end{gathered}
$$

which are close, in the approprate sense, to the basic solution $Z_{a}=A_{a}$. We identify solutions which differ by the gauge transformation $Z_{a} \mapsto g^{-1} Z_{a} g, g \in U(\mathcal{H})$.

Now let us count the solutions $(102)$ equivariantly with respect to the maximal torus $\mathbb{T}$ of $S U(4)$ which acts linearly on the $Z_{a}$ 's:

$$
Z_{a} \mapsto \sum_{b \in \underline{4}} u_{a}^{b} Z_{b} \quad u u^{\dagger}=1, \quad \operatorname{det}(u)=1
$$


The basic solution is invariant under 103 in the sense that such a transformation can be undone by the unitary similarity transformation. Infinitesimally:

$$
\varepsilon_{a} A_{a}=\left[\Phi_{0}, A_{a}\right]
$$

where

$$
\Phi_{0}=\bigoplus_{a<b \in \underline{4}} \operatorname{diag}\left(\mathfrak{a}_{a b, \alpha}\right)_{\alpha \in\left[n_{a b}\right]} \otimes 1_{\mathcal{H}_{2}}+1_{N_{a b}} \otimes\left(\varepsilon_{a} \mathbf{a}_{1}^{\dagger} \mathbf{a}_{1}+\varepsilon_{b} \mathbf{a}_{2}^{\dagger} \mathbf{a}_{2}\right)
$$

The infinite-dimensional version of the fixed point localization technique would reduce the counting to the enumeration of the solutions which are invariant, modulo gauge transformations:

$$
\varepsilon_{a} Z_{a}=\left[\Phi, Z_{a}\right]
$$

where $\Phi-\Phi_{0}$ is a compact self-adjoint operator in $\mathcal{H}$, with the eigenvalues of the form $\mathfrak{a}_{a b, \alpha}+\varepsilon_{a} i_{a}+\varepsilon_{b} i_{b}$, with $i_{1}, i_{2}, i_{3}, i_{4} \in \mathbb{Z}, \alpha=1, \ldots, n_{a b}=\operatorname{dim} N_{a b}$.

The fixed point contribution, including the perturbative prefactor is given by the ratio of regularized determinants:

$$
\frac{\operatorname{Det}^{\prime}(\operatorname{ad}(\Phi)) \operatorname{Pf}\left(\prod_{a<b}\left(\operatorname{ad}(\Phi)+\varepsilon_{a}+\varepsilon_{b}\right)\right)}{\prod_{a} \operatorname{Det}\left(\operatorname{ad}(\Phi)+\varepsilon_{a}\right)} \sim \epsilon\left[-P_{123} H H^{*}\right],
$$

where $P_{123}=\left(1-q_{1}\right)\left(1-q_{2}\right)\left(1-q_{3}\right), q_{a}=e^{\tau \varepsilon_{a}}, H=\operatorname{Tr}_{\mathcal{H}} e^{\tau \Phi}, H^{*}=\operatorname{Tr}_{\mathcal{H}} e^{-\tau \Phi}$, etc. To arrive at 79. one imposes additional $\Gamma_{\gamma^{-}}$-orbifold projections, as in [97, 98].

\section{Three types of surface defects}

In this section we discuss three types of surface defects: the ones defined using the orbifold of the spacetime by a cyclic group; the ones obtained by coupling the theory to the nearly Higgsed gauge theory, we call it a quiver construction for the reasons which will become clear below; and the folded defects, which correspond to the gauge theory on singular spacetime, a product of a smooth Riemann surface and a nodal curve. The connection between the first two types of defects has been conjectured long time ago, and recently has been discussed in the context of the renormalization theory in [40. The rigorous results were obtained in 67 . leading to the proof of the conjectures of [95].

\subsection{The orbifold construction}

The operators defined by the orbifold projection were studied earlier in [4, 6, 8, 22, 90. We give the geometric realization of these operators below.

We start by recalling the ADHM construction: consider the moduli space $\boldsymbol{\prod}_{k, n}$ of matrices $\left(B_{1}, B_{2}, I, J\right), B_{1}, B_{2} \in \operatorname{End}(K), I \in \operatorname{Hom}(N, K), J \in \operatorname{Hom}(K, N)$, obeying the following equations and stability conditions:

$$
\begin{aligned}
& {\left[B_{1}, B_{2}\right]+I J=0} \\
& \text { Stability: } \mathbb{C}\left[B_{1}, B_{2}\right] I(N)=K
\end{aligned}
$$


modulo the symmetry

$$
\left(B_{1}, B_{2}, I, J\right) \longrightarrow\left(g^{-1} B_{1} g, g^{-1} B_{2} g, g^{-1} I, J g\right), \quad g \in G L(K)
$$

The supersymmetric partition function of $\mathcal{N}=2$ supersymmetric gauge theory on $\mathbb{R}^{4} \approx \mathbb{C}^{2}$ with appropriate supersymmetric boundary conditions at infinity, with the gauge group $U(n)$, in the instanton sector $k$, localizes onto the integral of an equivariant differential form on $\prod_{k, n}$. More precisely, in defining $\boldsymbol{\Pi n}_{k, n}$ we used a particular stability condition in (108). It actually corresponds to deforming $\mathbb{R}^{4}$ into a noncommutative space $\mathbb{R}_{\theta}^{4}$, such that the holomorphic coordinates $z_{1}, z_{2}$ remain commuting, while their commutators with the conjugates obey: $\left[z_{1}, \bar{z}_{1}\right]+\left[z_{2}, \bar{z}_{2}\right]=-\zeta, \zeta>0$.

Now let us insert a surface operator along the $\mathbb{R}^{2} \approx \mathbb{C}^{1}$ plane, with the coordinate $z_{1}$, i.e. the surface is defined by the equation $z_{2}=0$.

Motivated by [15, 16, 42] we perform the orbifold, following the [32] construction: make $N$ and $K$ the $\mathbb{Z}_{p}$-modules:

$$
N=\bigoplus_{\nu} N_{\nu} \otimes \mathcal{R}_{\nu}, \quad K=\bigoplus_{\nu} K_{\nu} \otimes \mathcal{R}_{\nu}
$$

with $\mathcal{R}_{\boldsymbol{\nu}}, \boldsymbol{\nu}=0,1, \ldots, p-1, \boldsymbol{\nu}+p \equiv \boldsymbol{\nu}$, being the one-dimensional irreducible representation of $\mathbb{Z}_{p}$, where the generator $\omega$ acts via:

$$
T_{\mathcal{R}_{\nu}}(\omega)=\omega^{\nu} \equiv e^{\frac{2 \pi \mathrm{i} \nu}{p}}
$$

and impose the equivariance condition, with $\Omega_{K}=T_{K}(\omega), \Omega_{N}=T_{N}(\omega)$ :

$$
B_{1}=\Omega_{K}^{-1} B_{1} \Omega_{K}, \quad \omega B_{2}=\Omega_{K}^{-1} B_{2} \Omega_{K}, \quad I=\Omega_{K}^{-1} I \Omega_{N} \quad \omega J=\Omega_{N}^{-1} J \Omega_{K}
$$

which imply that the operators $B_{1}, B_{2}, I, J$ decompose as $B_{1}=\left(B_{1, \nu}\right)_{\nu}, B_{2}=\left(B_{2, \nu}\right)_{\nu}, I=\left(I_{\nu}\right)_{\nu}$, $J=\left(J_{\nu}\right)_{\nu}$, with

$$
\begin{gathered}
B_{1, \nu}: K_{\nu} \rightarrow K_{\nu}, B_{2, \nu}: K_{\nu} \rightarrow K_{\nu-1} \\
I_{\nu}: N_{\nu} \rightarrow K_{\nu}, J_{\nu}: K_{\nu} \rightarrow N_{\nu-1}
\end{gathered}
$$

The projected equations

$$
B_{1, \nu-1} B_{2, \nu}-B_{2, \nu} B_{1, \nu}+I_{\nu-1} J_{\nu}=0
$$

Now use the identity:

$$
\left[B_{1}, B_{2}^{p}\right]=\sum_{m=0}^{p-1} B_{2}^{m}\left[B_{1}, B_{2}\right] B_{2}^{p-1-m}=-\sum_{m=0}^{p-1} B_{2}^{m} I J B_{2}^{p-1-m}
$$

to arrive at:

$$
\left[\tilde{B}_{1}, \tilde{B}_{2}\right]+\tilde{I} \tilde{J}=0
$$

where $\tilde{B}_{1}, \tilde{B}_{2} \in \operatorname{End}(\tilde{K}), \tilde{I} \in \operatorname{Hom}(\tilde{N}, \tilde{K}), \tilde{J} \in \operatorname{Hom}(\tilde{K}, \tilde{N})$ are the new ADHM matrices, with

$$
\begin{aligned}
& \tilde{K}=K_{p-1}, \quad \tilde{N}=\bigoplus_{\nu} N_{\nu}, \\
& \tilde{B}_{1}=B_{1, p-1}, \quad \tilde{B}_{2}=B_{2,0} B_{2,1} \ldots B_{2, p-1}, \\
& \tilde{I}=\sum_{m=0}^{p-1} B_{2,0} B_{2,1} \ldots B_{2, m-1} I_{m-1}, \quad \tilde{J}=\sum_{m=0}^{p-1} J_{m} B_{2, m+1} \ldots B_{2, p-1},
\end{aligned}
$$


We thus get a map: $\boldsymbol{n}_{k, n}^{\text {orb }} \rightarrow \boldsymbol{\pi}_{\tilde{k}, n}$. Integrating along the fibers of this map we produce a cohomology class of $\prod_{\tilde{k}, n}$ which one can express in terms of the equivariant Donaldson classes.

Let us now give the explicit expression of this class, the representative of the corresponding surface operator in the $\mathcal{Q}$-cohomology of the gauge theory in the $\Omega$-background. We do it in two examples, the $A_{1}$ theory, i.e. the $U(N)$ theory with $2 N$ fundamental hypermultiplets, and the $\mathcal{N}=2^{*}$ theory.

In the both cases the first step is to perform the $\mathbb{Z}_{p}$-projection of the vector multiplet contribution to the instanton measure. To save the space we omit the explicit mention of the $\tau$-dependence, and denote by $*$ the $\tau \rightarrow-\tau$ conjugation, as well as $\mathcal{R}_{\nu}^{*}=\mathcal{R}_{p-\nu}$ :

$$
\epsilon\left[-\sum_{\boldsymbol{\nu}, \boldsymbol{\nu}^{\prime \prime}=0}^{p-1} \frac{S_{\boldsymbol{\nu}^{\prime \prime}} S_{\boldsymbol{\nu}}^{*}}{P_{1}\left(1-\tilde{q}_{2}\right)} \sum_{\boldsymbol{\nu}^{\prime}=0}^{p-1} q_{2}^{\boldsymbol{\nu}^{\prime}} \delta_{\boldsymbol{\nu}^{\prime \prime}+\boldsymbol{\nu}^{\prime}-\boldsymbol{\nu}}^{\mathbb{Z}_{p}}\right]
$$

where $P_{1}=1-q_{1}, \tilde{q}_{2}=q_{2}^{p}$ and we used the identity:

$$
\frac{1}{1-q_{2} \mathcal{R}_{1}}=\frac{1}{1-\tilde{q}_{2}} \sum_{\nu=0}^{p-1} q_{2}^{\nu} \mathcal{R}_{\nu}
$$

in $K\left[\mathbb{Z}_{p}\right] \otimes \mathbb{C}$. Define

$$
\begin{gathered}
\tilde{S}_{\boldsymbol{\nu}}=S_{\boldsymbol{\nu}} q_{2}^{-\boldsymbol{\nu}}=\tilde{N}_{\boldsymbol{\nu}}-P_{1} \tilde{K}_{\boldsymbol{\nu}}+P_{1}\left\{\begin{array}{l}
\tilde{K}_{\boldsymbol{\nu}-1}, \boldsymbol{\nu}>0 \\
\tilde{K}_{p-1} \tilde{q}_{2}, \boldsymbol{\nu}=0
\end{array},\right. \\
\tilde{S}=\sum_{\boldsymbol{\nu}=0}^{p-1} \tilde{S}_{\boldsymbol{\nu}}=\tilde{N}-P_{1} \tilde{P}_{2} \tilde{K}
\end{gathered}
$$

where $\tilde{P}_{2}=1-\tilde{q}_{2}$. Now we can rewrite 119$)$ as

$$
\epsilon\left[-\frac{\tilde{S} \tilde{S}^{*}}{P_{1} \tilde{P}_{2}}\right] \cdot \epsilon\left[\frac{1}{P_{1}} \sum_{0 \leq \nu^{\prime}<\nu^{\prime \prime}<p} \tilde{S}_{\nu^{\prime \prime}} \tilde{S}_{\nu^{\prime}}^{*}\right]
$$

The first factor is the usual four dimensional vector multiplet instanton measure, while the second is the two dimensional gauged linear sigma model contribution, corresponding to the sigma model on the (partial) flag variety, cf. (83).

Let us clarify the latter statement. At the level of fixed points, the $N$-tuples of Young diagrams $\lambda^{(\alpha)}, \alpha=1, \ldots, N$ enumerate the fixed points of the $U(1)^{N} \times U(1) \times U(1)$-action both on $\pi_{k, n}^{\text {orb }}$ and on $\pi_{\tilde{k}, n}$. However, the map is many-to-one. Let $c:[N] \rightarrow\{0,1, \ldots, p-1\}$ be the coloring function, which describes the decomposition (110):

$$
N_{\nu}=\sum_{\alpha \in c^{-1}(\nu)} e^{\tau a_{\alpha}}
$$

Then

$$
K_{\nu}=\sum_{\alpha=1}^{N} e^{\tau a_{\alpha}} \quad \sum_{i=1}^{\left(\lambda^{(\alpha)}\right)^{t}} q_{1}^{i-1} \sum_{1 \leq j \leq \lambda_{i}^{(\alpha)}, j-1+c(\alpha) \equiv \nu(p)} q_{2}^{j-1}
$$


Let

$$
\tilde{a}_{\alpha}=a_{\alpha}-\varepsilon_{2} c(\alpha)
$$

so that

$$
\begin{gathered}
\tilde{N}_{\boldsymbol{\nu}}=\sum_{\alpha \in c^{-1}(\boldsymbol{\nu})} e^{\tau \tilde{a}_{\alpha}}, \\
\tilde{K}_{\nu}=K_{\nu} q_{2}^{-\nu}=\sum_{\alpha=1}^{N} e^{\tau \tilde{a}_{\alpha}} \sum_{i=1}^{\left(\lambda^{(\alpha)}\right)^{t}} q_{1}^{i-1} \sum_{1 \leq j \leq \lambda_{i}^{(\alpha)}, j-1+c(\alpha) \equiv \nu(p)} \tilde{q}_{2}^{\frac{j-1+c(\alpha)-\boldsymbol{\nu}}{p}}= \\
\sum_{\alpha=1}^{N} e^{\tau \tilde{a}_{\alpha}} \sum_{i=1}^{\left(\lambda^{(\alpha)}\right)^{t}} q_{1}^{i-1} \sum_{j=1 \text { or } 2}^{l_{\alpha, i, \boldsymbol{\nu}}} \tilde{q}_{2}^{j-1}
\end{gathered}
$$

where $l_{\alpha, i, \boldsymbol{\nu}}=\left[\frac{\lambda_{i}^{(\alpha)}+c(\alpha)-\boldsymbol{\nu}+p-1}{p}\right]$, and the lower limit of the sum over $j$ is equal to 1 for $c(\alpha) \leq \boldsymbol{\nu}$ and 2 otherwise. We can also write, in terms of the dual partitions $\lambda^{(\alpha) t}$ :

$$
P_{1} \tilde{K}_{\nu}=\sum_{\alpha=1}^{N} e^{\tilde{a}_{\alpha}} \sum_{J=1 \text { or } 2} \tilde{q}_{2}^{J-1}\left(1-q_{1}^{\lambda_{1-c(\alpha)+p(J-1)+\nu}^{(\alpha) t}}\right)
$$

where, again, $J \geq 1$ when $\boldsymbol{\nu} \geq c(\alpha)$, and $J \geq 2$ when $\boldsymbol{\nu}<c(\alpha)$.

Now, for $\boldsymbol{\nu}=p-1$ :

$$
\tilde{K}=\sum_{\alpha=1}^{N} e^{\tau \tilde{a}_{\alpha}} \sum_{i=1}^{\left(\Lambda^{(\alpha)}\right)^{t}} q_{1}^{i-1} \sum_{j=1}^{\Lambda_{i}^{(\alpha)}} \tilde{q}_{2}^{j-1}
$$

where

$$
\Lambda_{i}^{(\alpha)}=\left[\frac{\lambda_{i}^{(\alpha)}+c(\alpha)}{p}\right]
$$

We denote by $\rho_{c}$ the map of the set of $N$-tuples of partitions to itself, given by (131):

$$
\left(\Lambda^{(\alpha)}\right)=\rho_{c}\left(\lambda^{(\alpha)}\right)
$$

The map $\rho_{c}$ is, in general, $\infty: 1$. Indeed, any $\lambda_{i}^{(\alpha)}<p-c(\alpha)$ for sufficiently large $i$ will map to $\Lambda_{i}^{(\alpha)}=0$. There is no room for such ambiguity for $\alpha \in c^{-1}(p-1)$.

Let

$$
k_{\nu}=\left|K_{\nu}\right|=\sum_{\alpha=1}^{N} \sum_{i=1}^{\left(\lambda^{(\alpha)}\right)^{t}}\left(\left[\frac{\lambda_{i}^{(\alpha)}+c(\alpha)-\nu+p-1}{p}\right]-\left[\frac{c(\alpha)-\boldsymbol{\nu}+p-1}{p}\right]\right)
$$

In particular

$$
\tilde{k}=k_{p-1}=\sum_{\alpha=1}^{N}\left|\Lambda^{(\alpha)}\right|
$$


Define the auxiliary variables $\left(z_{\boldsymbol{\nu}}\right)$ and $\mathfrak{q}$, via

$$
\mathfrak{q}_{0}=z_{1} / z_{0}, \mathfrak{q}_{1}=z_{2} / z_{1}, \ldots, \mathfrak{q}_{p-1}=\mathfrak{q} z_{0} / z_{p-1}
$$

Define $\Sigma_{0}=0, \Sigma_{p}=\tilde{S}$ and $\Sigma_{\boldsymbol{\nu}}, \boldsymbol{\nu}=1, \ldots, p-1$, via:

$$
\tilde{S}_{\nu}=\Sigma_{\nu+1}-\Sigma_{\nu}
$$

so that

$$
\Sigma_{\nu}=\tilde{N}_{0}+\tilde{N}_{1}+\ldots+\tilde{N}_{\nu-1}-P_{1} \tilde{K}_{\nu-1}+\tilde{q}_{2} P_{1} \tilde{K}
$$

Thus, the surface defect, as an operator of the four dimensional chiral ring, is given by:

$$
\mathcal{I}_{\Lambda^{(\alpha)}}=\sum_{\left(\lambda^{(\alpha)}\right) \in \rho_{c}^{-1}\left(\Lambda^{(\alpha)}\right)} \prod_{\nu=0}^{p-1} z_{\nu}^{k_{\nu-1}-k_{\nu}} \cdot \epsilon\left[\frac{1}{P_{1}} \sum_{\nu=1}^{p-1}\left(\Sigma_{\nu+1}-\Sigma_{\nu}\right) \Sigma_{\nu}^{*}\right]
$$

This is the partition function of the two dimensional gauged linear sigma model on the partial flag variety $F \operatorname{lags}\left(d_{1}, d_{2}, \ldots, N\right)$, with

$$
d_{\nu}=\#\{\alpha \mid c(\alpha)<\nu\},
$$

coupled to the four dimensional gauge theory, via $\Sigma_{p}=\tilde{S}$.

This would be the end of the story for the pure super-Yang-Mills theory. In particular, for regular defect, $p=N$, with $d_{\nu}=\boldsymbol{\nu}$, we get the setup of [19]. However, the power of our approach is the ability to include the matter fields. In the $A_{1}$ case the orbifold projection must also include the decomposition of the matter multiplet:

$$
M=\bigoplus_{\nu=0}^{p-1} M_{\nu} \otimes \mathcal{R}_{\nu}
$$

The additional factor in the instanton measure is

$$
\epsilon\left[-\sum_{\nu} \tilde{M}_{\nu}^{*} \tilde{K}_{\nu}\right]=\epsilon\left[-\tilde{M}^{*} \tilde{K}\right] \cdot \epsilon\left[-\frac{1}{P_{1}} \sum_{\nu=0}^{p-1} \tilde{M}_{\nu}^{*}\left(\tilde{N}_{0}+\tilde{N}_{1}+\ldots+\tilde{N}_{\nu}-\Sigma_{\nu+1}\right)\right]
$$

where

$$
\tilde{M}=\tilde{q}_{2}^{-1} \sum_{\nu} \tilde{M}_{\nu}
$$

which means that the surface operator is now the partition function of the two dimensional sigma model with the target space the total space of the vector bundle over $\operatorname{Flags}\left(d_{1}, d_{2}, \ldots, d_{p-1}, N\right)$ with the fiber

$$
\bigoplus_{i=1}^{p-1} \operatorname{Hom}\left(\mathcal{E}_{i}, \tilde{M}_{i-1}\right)
$$

where $\mathcal{E}_{i}, r k \mathcal{E}_{i}=d_{i}$ is the $i$ 'th tautological bundle. 
In the $\mathcal{N}=2^{*}$ case the instanton measure is

$$
\epsilon\left[-P_{3} \sum_{\boldsymbol{\nu}, \boldsymbol{\nu}^{\prime \prime}=0}^{p-1} \frac{S_{\boldsymbol{\nu}^{\prime \prime}} S_{\boldsymbol{\nu}}^{*}}{P_{1}\left(1-\tilde{q}_{2}\right)} \sum_{\boldsymbol{\nu}^{\prime}=0}^{p-1} q_{2}^{\boldsymbol{\nu}^{\prime}} \delta_{\boldsymbol{\nu}^{\prime \prime}+\boldsymbol{\nu}^{\prime}-\boldsymbol{\nu}}^{\mathbb{Z}_{p}}\right]
$$

with $P_{3}=1-q_{3}$, which gives, for the surface operator

$$
\mathcal{I}_{\Lambda^{(\alpha)}}=\sum_{\left(\lambda^{(\alpha)}\right) \in \rho_{c}^{-1}\left(\Lambda^{(\alpha)}\right)} \prod_{\nu=0}^{p-1} z_{\nu}^{k_{\nu^{-1}}-k_{\nu}} \cdot \epsilon\left[\frac{P_{3}}{P_{1}} \sum_{\nu=1}^{p-1}\left(\Sigma_{\nu+1}-\Sigma_{\nu}\right) \Sigma_{\nu}^{*}\right]
$$

which corresponds to the softly broken $\mathcal{N}=(4,4)$ sigma model, with the hyperkähler target space $T^{*} F \operatorname{lags}\left(d_{1}, d_{2}, \ldots, d_{p-1}, N\right)$. The mass $\varepsilon_{3}$ of the adjoint hypermultiplet translates to the twisted mass corresponding to the $U(1)$ symmetry of the cotangent fibers: $t=q_{2} q_{4}$.

\subsection{The quiver construction}

Consider the $A_{2}$ quiver gauge theory, with the gauge group $U(N) \times U(N)$, and $N$ fundamental hypermultiplets at either node of the quiver:

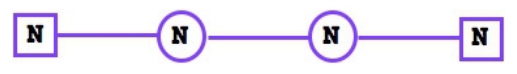

The theory depends on $2 N$ masses $m_{f}^{ \pm}, f=1, \ldots, N$ and $2 N$ Coulomb parameters $\mathfrak{a}_{\alpha}^{ \pm}$, $\alpha=1, \ldots, N$. This theory can be obtained by taking a limit $\mathfrak{q}_{0}, \mathfrak{q}_{3} \rightarrow 0$ of the $\hat{A}_{3}$-type quiver theory, which corresponds to the $\mathbb{Z}_{4}$ orbifold of the 3,4-space. Its partition function is given by the sum over $2 N$-tuples of Young diagrams $\lambda^{( \pm, \alpha)}, \alpha=1, \ldots, N$. The contribution of a specific $2 \mathrm{~N}$-tuple of Young diagrams is given by:

$$
\begin{aligned}
\epsilon\left[\sum_{a= \pm} N_{a} K_{a}^{*}+N_{a}^{*} K_{a} q_{12}-P_{12} K_{a} K_{a}^{*}\right] \times & \\
& \in\left[-M_{+} K_{+}^{*}-N_{+} K_{-}^{*}-q_{12} K_{+} N_{-}^{*}+P_{12} K_{+} K_{-}^{*}-K_{-} M_{-}^{*}\right]
\end{aligned}
$$

where

$$
\begin{aligned}
N_{a}^{(\tau)} & =\sum_{\alpha=1}^{N} e^{\tau \mathfrak{a}_{\alpha}^{a}}, \quad M_{a}=\sum_{f=1}^{N} e^{\tau m_{f}^{a}} \\
K_{a} & =\sum_{\alpha=1}^{N} e^{\tau \mathfrak{a}_{\alpha}^{a}} \sum_{(i, j) \in \lambda^{(a, \alpha)}} q_{1}^{i-1} q_{2}^{j-1}
\end{aligned}
$$

with $q_{1,2}=e^{\tau \varepsilon_{1,2}}$.

Let us consider the special arrangement of masses and Coulomb parameters:

$$
N_{+}=M_{+} \Leftrightarrow \mathfrak{a}_{\alpha}^{+}=m_{\alpha}^{+}, \quad \alpha=1, \ldots, N
$$


The measure (146) vanishes unless $\lambda^{(+, \alpha)}=\emptyset$ for all $\alpha$. In this case it reduces to (with $K=K_{-}$):

$$
\epsilon\left[N K^{*}+N^{*} K q_{12}-P_{12} K K^{*}-M_{+} K^{*}-K M_{-}^{*}\right]
$$

which is the measure of the $A_{1}$ theory, also known as $U(N)$ theory with $2 N$ fundamental hypermultiplets with the masses $m_{f}^{ \pm}$. This reduction is most simply explained using the description of the moduli space of quiver instantons [97]: the identification $M_{+}=N_{+}$ means that we identify the vector spaces $\mathbf{M}_{+}$and $\mathbf{N}_{+}$, thereby making the obstruction bundle $\operatorname{Hom}\left(\mathbf{M}_{+}, K_{+}\right)=\operatorname{Hom}\left(\mathbf{N}_{+}, K_{+}\right)$. The latter has the equivariant section $I_{+}: \mathbf{N}_{+} \rightarrow K_{+}$. Its zero locus is the space of the quiver ADHM data $\left(B_{1,2, \pm}, I_{ \pm}, J_{ \pm}, \ldots\right)$ for which $I_{+}=0$. Since (cf. [97]) $K_{+}=\mathbb{C}\left[B_{1,+}, B_{2,+}\right] I_{+}\left(\mathbf{N}_{+}\right)$(in the stability chamber where all $\zeta_{a}^{\mathbf{R}}>0$ ) we get $K_{+}=0$.

Now let us impose a less stringent relation between $M_{+}$and $N_{+}$:

$$
M_{+}=N_{+}-P_{2} \mu
$$

where $\mu=e^{\tau\left(m_{f}^{+}-\varepsilon_{2}\right)}$ for one of the $f$ 's. The equivariant section $I_{+}$now modifies to :

$$
B_{2,+} I_{+} \Pi_{f}+I_{+}\left(1-\Pi_{f}\right): \mathbf{N}_{+} \longrightarrow K_{+}
$$

where $\Pi_{f}: N_{+} \rightarrow N_{+}$is the projection onto the eigenvector of $g_{\text {flavor }}$ with the eigenvalue $\mu$.

The fixed point locus in this case allows for nontrivial $K_{+}$, however, only the row type Young diagrams are allowed:

$$
K_{+}=\mu \frac{1-q_{1}^{k}}{1-q_{1}}
$$

for some $k \geq 0$. The instanton measure (146) simplifies to

$$
\epsilon\left[\mathcal{N} K^{*}+\mathcal{N}^{*} K q_{12}-P_{12} K K^{*}-\mathcal{M}_{+} K^{*}-K \mathcal{M}_{-}^{*}\right] \times \mathcal{I}_{f}(K)
$$

with $K=K_{-}, \mathcal{N}=N_{-}, \mathcal{M}_{+}=N_{+}, \mathcal{M}_{-}=M_{-}$and, with $S=\mathcal{N}-P_{12} K$,

$$
\mathcal{I}_{f}(K)=\sum_{k=0}^{\infty} z^{k} \epsilon\left[P_{1} \mu q_{1}^{k} K_{+}^{*}+q_{12} K_{+}\left(\mathcal{M}_{+}-S\right)^{*}\right]=\sum_{k=0}^{\infty} \frac{k+1}{k !}\left(\frac{z}{\varepsilon_{1}}\right)^{k} \prod_{l=1}^{k} \frac{Y\left(m_{f}^{+}+\varepsilon_{1} l\right)}{\tilde{P}_{+}\left(m_{f}^{+}+\varepsilon_{1} l\right)}
$$

where $P_{+}(x)=\left(x-m_{f}^{+}\right) \tilde{P}_{+}(x)=\epsilon\left[-e^{\tau x} \mathcal{M}_{+}^{*}\right], Y(x)=\epsilon\left[-e^{\tau x} S^{*}\right]$, and $z=\mathfrak{q}_{+}$is the instanton fugacity of the + node of the $A_{2}$ quiver, which becomes the two dimensional sigma model Kähler modulus.

In fact, $\mathcal{I}_{f}(K)$ is the $I$-function [53, 54, 55] (which is often confused with the $J$-function, defined using the stable maps, i.e. the nonlinear sigma model as opposed to the quasimaps, i.e. the gauged linear sigma model). There are $N$ such functions, for $f=1, \ldots, N$. Together we should view them as the $H^{*}\left(\mathbb{C P}^{N-1}\right)$-valued function.

If we neglect the four dimensional instantons, i.e. replace $Y(x)$ by the Coulomb polynomial $A(x)=\prod_{i=1}^{N}\left(x-a_{i}\right)$, the partition function $(155)$ would be identified with the specification of (97) for the $\mathbb{C}^{N} \otimes \mathcal{O}(-1)$-bundle over $\mathbb{C P}^{N-1}$. Here $\mathbb{C}^{N}$ is the color space. In the present case, $\varepsilon_{1}$ is the relevant parameter of the $\Omega$-deformation. The gauge group of the effective sigma model is $U(1)$. The full expression $\mathcal{I}(K)$, which incorporates the effects of the four 
dimensional instantons (which generate the poles of $Y(x)$, making it the rational function of $x)$ can also be interpreted in the language of the two dimensional sigma model. However, the effective target space of the sigma model depends on the four dimensional gauge field configuration. In the non-equivariant limit $\varepsilon_{1}, \varepsilon_{2} \rightarrow 0$, (the limit shape [102]) the operator $\mathcal{I}_{f}(K)$ has the exponential asymptotics

$$
\mathcal{I}_{f}(K) \sim e^{\frac{\tilde{W}}{\varepsilon_{1}}}
$$

where $\tilde{W}$ is computed by extremizing:

$$
\log (z) \sigma+\int^{\sigma} \log \left(Y(x) / \tilde{P}_{+}(x)\right) d x
$$

It would be nice to compare this result with the conjectures of [45]. See also the further work on the surface defects and sigma models in [22, 90], and their three dimensional lifts in [71].

- One can study other types of arrangements of masses and Coulomb parameters leading to surface defects in the $A_{1}$ theory. For example, choose a subset $S \subset\{1, \ldots, N\},|S|=r$, and set

$$
M_{+}=N_{+}-P_{2} L, \quad L=\sum_{s \in S} e^{\tau a_{+, s}}
$$

Then,

$$
K_{+}=\sum_{s \in S} e^{\tau a_{+, s}} \frac{1-q_{1}^{k_{s}}}{1-q_{1}}
$$

The resulting surface defect operator will correspond to the $\left[\operatorname{Hom}\left(\mathcal{E}, M_{+}\right) \rightarrow \operatorname{Gr}(r, N)\right]$ sigma model coupled to the four dimensional gauge theory. The equivariant section is again given by the formula $(152)$ with $\Pi_{f}$ now the rank $r$ projection onto the corresponding subspace of $\mathbf{N}_{+}$.

The surface defects of this section were also recently studied in [111], [112].

\subsection{The folded construction}

Finally, we can get the surface defects with the help of the folded instanton construction [97, 98. Take the $U(N)$ gauge theory on the four dimensional space $\mathbb{C}_{12}^{2}$ and the $U(M)$ gauge theory on the four dimensional space $\mathbb{C}_{23}^{2}$. From the point of view of the observer living on the $\mathbb{C}_{12}^{2}$ space there are degrees of freedom propagating along the two dimensional surface $\mathbb{C}_{2}^{1}$, which are charged in the fundamental representation. In the $\Omega$-background with the parameters $\varepsilon_{1}, \varepsilon_{2}, \varepsilon_{3}, \varepsilon_{4}$, with

$$
\sum_{a=1}^{4} \varepsilon_{a}=0
$$

the degrees of freedom on the $\mathbb{C}_{23}^{2}$-space can be integrated out producing the effective surface operator:

$$
\sum_{K_{23}} \mathfrak{q}^{k_{23}} \epsilon\left[-\frac{S_{23}^{*} S_{23} P_{1}}{P_{23}^{*}}-\frac{S_{12} S_{23}^{*} q_{23}+S_{12}^{*} S_{23} q_{12}}{P_{2}}\right]
$$


If we freeze the bulk dynamics both in the $\mathbb{C}_{12}^{2}$ and the $\mathbb{C}_{23}^{2}$ spaces, the interaction 161 corresponds to the two dimensional $\mathcal{N}=(2,2)$ theory with two chiral bi-fundamental multiplets, one in the $(N, \bar{M})$ another in the $(\bar{N}, M)$ representation of $U(N) \times U(M)$, with the twisted masses given by $\varepsilon_{1}+\varepsilon_{2}$ and $\varepsilon_{2}+\varepsilon_{3}$, respectively.

One can also add the orbifold defects on top of the folded construction, with $\left(z_{2}, z_{4}\right) \mapsto$ $\left(\omega z_{2}, \omega^{-1} z_{4}\right)$. Then, effectively, we are getting a junction of two surface effects, one extended along the $\mathbb{C}_{1}^{1}$ plane, another along the $\mathbb{C}_{3}^{1}$ plane. This is a singular rational curve which can be deformed to the smooth curve. However, the deformation parameter has the equivariant weight $\varepsilon_{1}+\varepsilon_{3}$.

In the companion paper [99] will analyze these surface defects using the qq-characters. They are obtained by adding another component, e.g. $\mathbb{C}_{34}^{2}$, or $\mathbb{C}_{14}^{2}$, to the worldvolume of the gauge theory.

The compactness theorem of [97] is powerful when $\operatorname{Hom}_{\mathbb{Z}_{p}}\left(N_{12}, N_{34} \otimes \mathcal{R}_{1}\right) \approx \mathbb{C}$ and $\operatorname{Hom}_{\mathbb{Z}_{p}}\left(N_{34} \otimes\right.$

$\left.\mathcal{R}_{1}, N_{23}\right)=0$ for $\mathbb{C}_{34}^{2} q q$-operator, and, analogously $\operatorname{Hom}_{\mathbb{Z}_{p}}\left(N_{12}, N_{14} \otimes \mathcal{R}_{1}\right)=0$ and $\operatorname{Hom}_{\mathbb{Z}_{p}}\left(N_{14} \otimes\right.$ $\left.\mathcal{R}_{1}, N_{23}\right) \approx \mathbb{C}$ for the $\mathbb{C}_{14}^{2}$ operator. This is easy to arrange, e.g. for $p=N+M$. We shall study the corresponding KZ-like equations in [99].

\section{7 qq-characters in two dimensional sigma models}

In [94, 96, 97] we introduced the so-called qq-character observables in four dimensional $\mathcal{N}=2$ theories. They are convenient to express the non-perturbative Dyson-Schwinger equations.

In the two dimensional context one also expects the non-perturbative Dyson-Schwinger equations to take place and to be useful. We shall be working in the softly broken $\mathcal{N}=(4,4)$ quiver gauge theory case.

Define, for $i \in \mathrm{V}_{\gamma}$,

$$
Y_{i}(x)=\frac{Q_{i}(x)}{Q_{i}(x-2 u)}, \quad D_{i}(x)=\frac{P_{i}(x+u)}{P_{i}(x-u)}
$$

We shall view $Q_{i}(x)$ and $Y_{i}(x)$ as the vacuum expectation values of the observables $\mathbf{Q}_{i}(x)$ and $\mathbf{Y}_{i}(x)$ :

$$
\mathbf{Q}_{i}(x)=\operatorname{Det}_{\mathbf{N}_{i}}\left(x-\sigma_{i}\right), \quad \mathbf{Y}_{i}(x)=\frac{\mathbf{Q}_{i}(x)}{\mathbf{Q}_{i}(x-2 u)}
$$

Then (86) reads:

$$
Y_{i}(\xi+2 u)=-\tilde{\mathfrak{q}}_{i} \frac{D_{i}(\xi)}{Y_{i}(\xi)} \prod_{e \in t^{-1}(i)} Y_{s(e)}\left(\xi+u+m_{e}\right) \prod_{e \in s^{-1}(i)} Y_{t(e)}\left(\xi+u-m_{e}\right)
$$

for all $\xi$, s.t. $Q_{i}(\xi)=0$, with

$$
\tilde{\mathfrak{q}}_{i}=\mathfrak{q}_{i}(-1)^{m_{i}+n_{i}-1} \prod_{j \in \mathrm{V}_{\gamma}}(-1)^{C_{i j} n_{j}}
$$

The relations (164) can be interpreted as the reflections generating the generalization of the Weyl group $\mathcal{W}_{\Gamma}$ [103, 104]. The natural step is to introduce the Weyl-invariant, which is characterized by the property that the only poles in $x$ is has come from the poles of $P_{i}$ 's, i.e. 
all the superficial poles coming from $Q_{i}$ 's cancel between themselves. Thus, for every $i \in \mathrm{V}_{\gamma}$ we define:

$$
\mathbf{T}_{i}(x)=\mathbf{Y}_{i}(x+2 u)+\tilde{\mathfrak{q}}_{i} \frac{D_{i}(x)}{\mathbf{Y}_{i}(x)} \prod_{e \in t^{-1}(i)} \mathbf{Y}_{s(e)}\left(x+u+\mu_{e}\right) \prod_{e \in s^{-1}(i)} \mathbf{Y}_{t(e)}\left(x+u-\mu_{e}\right)+\ldots
$$

As we recalled above, for affine $A D E$ quivers $\gamma$ the two dimensional quiver gauge theory can be engineered as the low energy limit of the theory on a stack of fractional $D 1$ strings [32] localized at the tip 0 of the $A D E$ orbifold singularity $0 \in \mathbb{C}^{2} / \Gamma_{\gamma}$. As we explained in [96, 97, 98, 105] the observable (166) is the result of integrating out the open strings, connecting these branes and the stack of (fractional) D3-brane wrapping the ALE space $\mathbb{C}^{2} / \Gamma_{\gamma}$.

The full expression for the qq-character (166) is identical to that given in [96], the sum of integrals over the Nakajima varieties corresponding to the quiver $\gamma$. It makes sense for all quivers, not just the ADE type. The difference between the two dimensional and the four dimensional cases is the nature of the $\mathbf{Y}_{i}(x)$-observables and the $D_{i}(x)$-operators. In two dimensions both $\mathbf{Y}_{i}(x)$ and $D_{i}(x)$ are degree zero ratios of polynomials, as in (162). In four dimensions [96] $D_{i}(x)$ are the polynomials whose roots are the masses of the fundamental hypermultiplets, while $Y_{i}(x)=\epsilon\left[-e^{x} S_{i}^{*}\right]$ is the value of $\mathbf{Y}_{i}(x)$ in the instanton background, it is a degree $n_{i}$ rational function of $x$.

The qq-character is a local observable in both two and four dimensional theories. By exploiting the analytic properties of its expectation values one can derive numerous Ward identities.

In the limit $\varepsilon \rightarrow 0$ the $q q$-character becomes the $q$-character (more precisely, its Yangian version). The algebraic theory of $q$-characters was introduced in [41]. Their physical use was found first in [104] in the applications to the four and five dimensional $\mathcal{N}=2$ theories subject to the two-dimensional $\Omega$-deformation.

\section{Conclusions and outlook}

Here are some topics we have left out.

We discussed two dimensional supersymmetric gauge theories coupled to the four dimensional ones. In the quiver construction the two dimensional theory arises as the effective description of the four dimensional theory, similar to the cosmic string theory to which the $\mathcal{N}=2$ theory reduces with the special mass deformation which vanishes along a complex curve [122. Here the role of the mass deformation is played by the Higgsing of the gauge group (more precisely the gauge group factor) in the presence of $\Omega$-deformation. It would be nice to have a more detailed field theoretic description of this Higgsing and the generation of the mass gap which vanishes along a codimension two surface. Perhaps the noncommutative description of the section 5 could be used in this regard. See also [117] for the similar string solutions.

One should note that there is a simpler version of this construction, where the whole four dimensional theory becomes effectively the two dimensional one. Of course, any four dimensional theory can be viewed as a two dimensional theory with an infinite set of fields. Here we talk about the equivalence of the four dimensional theory and conventional two dimensional field theory. See [25, 33, 34] for numerous examples. 
The qq-characters which we discussed in the section 6 can be used to give a two dimensional gauge theory realization of the integrable lattice systems built using the $R$-matrices of the Yangians (or quantum affine algebras). This realization is used in the explanations 4 of the duality relating the Bethe/gauge correspondence and the recently revived four dimensional version of Chern-Simons theory [5, 61, 91, 26, 27, 124.

The moduli space of vortex solutions in the two dimensional theory on the surface of defect coupled to the four dimensional instantons can be given an ADHM-like description, by taking the quiver instanton moduli space [97] and imposing the additional equations (152). It would be interesting to compare the resulting ADHM-like equations to those in [21]. Also, our moduli spaces require the four dimensional ADHM-like data. The (Grassmanian) sigma models can be described by fewer degrees of freedom. The connection between these two description is, perhaps, the manifestation of the renormalization group flow argued in [40].

There is an interesting class of local observables in supersymmetric gauge theory, which are obtained by lifting the (partially) twisted theory on the blowup $\pi: \hat{X}_{p} \rightarrow X$ of the spacetime at a point $p$, and integrating out the degrees of freedom associated with the exceptional divisor $\pi^{-1}(p)$. Using the quiver description of the moduli space of instantons on the blowup [70, 87, 88] one can express the resulting operator as a series in the Y-observables, similar to (155). Also, by comparing the $\Omega$-deformed theories with the surface defects on the blowup to the original theory one can derive a set of Hirota-like relations involving the surface defect partition function and the bulk partition function. These relations explain the results of [47].

It would be nice if the folded construction could be used to derive the differential/difference equations obeyed by the partition functions (79), connecting to the results of [82, [109] (see [72] where such equations are derived, for the $A$-type quivers, using the methods of [82]).

The orbifold defects we studied in the four dimensional gauge theory can also be defined in the two dimensional gauged linear sigma models. In certain cases these produce the wavefunctions of the Bethe states of the quantum integrable systems dual to the $\mathcal{N}=(2,2)$ theory via Bethe/gauge correspondence [100, 23, 58. However, these constructions require the theory to have a large flavor symmetry. It would be nice to understand the results of [1] in these terms. More generally, the stable envelope basis of [82] should translate to the canonical basis in the space of supersymmetric codimension two defects.

We mostly talked about the two dimensional defects within four dimensional theories. Everything we said, including the qq-characters (hence the name) lifts to the three-dimensional defects within five dimensional theories, compactified on a circle of radius $R$ (see, e.g. [71]). One can get new observables in four dimensional theories not only by sending the radius $R$ back to zero, but also by considering the extreme limits of the orbifold defects we discussed. In particular, the $p \rightarrow \infty$ limit of the $\mathbb{Z}_{p}$ orbifold defect in the theory on $\mathbb{R}^{4} \times \mathbf{S}_{R}^{1}$ becomes a supersymmetric boundary condition in the four dimensional theory on $\mathbb{R}^{2} \times \mathbb{R}_{+}^{1} \times \mathbf{S}_{R}^{1}$. Another useful construction involves the blowup-type defects modeled on the ALE singularity $\mathbb{C}^{2} / \mathbb{Z}_{p}$. In the limit $p \rightarrow \infty$ the theory on $\mathbb{C}^{2} / \mathbb{Z}_{p} \times \mathbf{S}_{R}^{1}$ becomes the theory on $\mathbb{R}^{3} \times \mathbf{S}_{R}^{1}$ together with the monopole-like singularity at the origin in $\mathbb{R}^{3}=\mathbb{C}^{2} / U(1)$, corresponding to the 't Hooft line operator wrapping $\mathbf{S}_{R}^{1}$. In this way we get, essentially for free, the localization computation of the vacuum expectation values of the 't Hooft (and more general dyonic) operators in the $\mathcal{N}=2$ theory compactified on a circle. The computations in [64, 57, 24] confirm this. In this way one can hope to bring the conjectures of [46] within the reach of the conventional proofs.

\footnotetext{
${ }^{4}$ See the author's contribution to Strings-Math'2017 in Hamburg
} 
There are many more topics to explore and elucidate.

\section{Acknowledgements.}

Research was partly supported by the National Science Foundation under grant no. nsfphy/1404446. Any opinions, findings, and conclusions or recommendations expressed in this material are those of the authors and do not necessarily reflect the views of the National Science Foundation.

The author thanks A. Losev for discussions about two dimensional topological theories in 1992-1994, E. Frenkel for the invitation to the DARPA program 'Langlands Program and Physics' conference at the IAS in March 8-10 2004, and for the opportunity to present there some of the ideas developed in this paper, as well as for numerous patient explanations on $41]$ and other topics. The author is grateful to A. Okounkov, V. Pestun and A. Rosly for numerous useful discussions concerning the topics of this paper over the recent years, as well as to S. Jeong and O. Tsymbaliuk for the collaboration on the related projects.

The paper was finished while the author visited the IHES (Bures-sur-Yvette). We thank this remarkable institution for its hospitality.

\section{Appendix A. Defects in quantum mechanics}

Take two quantum systems, $\left(\hat{H}_{1}, \mathcal{H}_{1}\right)$ and $\left(\hat{H}_{2}, \mathcal{H}_{2}\right)$ with the Hilbert spaces $\mathcal{H}_{i}$ and the Hamiltonians $\hat{H}_{i}, i=1,2$. The spacetime manifolds $X_{1}$ and $X_{2}$ are, say, two intervals $X_{2}=\left[0, t_{2}\right]$, $X_{1}=\left[-t_{1}, 0\right]$, with $t_{1,2}>0$, the intersection $X_{12}$ consist of one point, $t=0$.

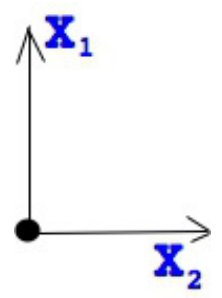

Then the path integral of the combined system computes:

$$
\left\langle\left.\psi_{2}\right|_{\mathcal{H}_{2}} e^{-\mathrm{i} t_{2} \hat{H}_{2}} \Pi_{12} e^{-\mathrm{i} t_{1} \hat{H}_{1}} \mid \psi_{1}\right\rangle_{\mathcal{H}_{1}}
$$

where $\Pi_{12}: \mathcal{H}_{1} \longrightarrow \mathcal{H}_{2}$ is an operator acting between the two Hilbert spaces, $\left|\psi_{1}\right\rangle_{\mathcal{H}_{1}} \in \mathcal{H}_{1}$, $\left|\psi_{2}\right\rangle_{\mathcal{H}_{2}}$ are the states of two systems, representing the boundary conditions at the other end of each interval. In the coordinate representation, the operator $\Pi_{12}$ has the kernel:

$$
\Pi_{12}\left(\phi_{1}, \phi_{2}\right)=\int[D \Psi] e^{-L_{12}\left(\Psi ; \phi_{1}, \phi_{2}\right)}
$$

where $\phi_{i}=\left.\boldsymbol{\Phi}_{i}\right|_{X_{12}}, i=1,2$. 
To make the example even more specific, although somewhat artificial, let us take the space $\mathcal{H}_{1}=\mathbb{C}\left[a_{1}^{\dagger}, \ldots, a_{p}^{\dagger}\right] \mid$ vac $\rangle$ of states of $p$ bosonic oscillators $\left[a_{i}, a_{j}^{\dagger}\right]=\delta_{i j}$, with the pseudovacuum $\mid$ vac $\rangle$ annihilated by $a_{i}$ 's, with the generic quadratic Hamiltonian

$$
\hat{H}_{1}=\sum_{i, j=1}^{p} K_{i j} a_{i}^{\dagger} a_{j}+B_{i j} a_{i} a_{j}+\bar{B}_{i j} a_{i}^{\dagger} a_{j}^{\dagger}
$$

with some matrices $K, B$ ( $B$ sufficiently small), and $\mathcal{H}_{2} \approx \mathbb{C}^{N}, N=2^{L}$, the space of states of $L$ fermionic oscillators $\left\{c_{A}, c_{B}^{\dagger}\right\}=\delta_{A B}$, with the Hamiltonian, say

$$
\hat{H}_{2}=\sum_{A, B=1}^{N} M_{A B} c_{A}^{\dagger} c_{B}+N_{A B} c_{A} c_{B}+\bar{N}_{B A} c_{A}^{\dagger} c_{B}^{\dagger}
$$

with some matrices $M, N$.

The operator $P_{12}$ orthogonally projects the space $\mathcal{H}_{1}$ onto the subspace of $\hat{Q}^{B}$ charge

$$
q=L+1-p
$$

states, which is then isomorphically mapped to the charge $\hat{Q}^{F}=q$ subspace of $\mathcal{H}_{2}$. Here the charges are defined by

$$
\hat{Q}^{B}=\sum_{i=1}^{p} a_{i}^{\dagger} a_{i}, \quad \hat{Q}^{F}=\sum_{A=1}^{L} c_{A}^{\dagger} c_{A}
$$

Explicitly:

$\Pi_{12}\left(\prod_{i=1}^{p} \frac{a_{i}^{n_{i}}}{\sqrt{n_{i} !}}|\mathrm{vac}\rangle\right)=\left\{\begin{array}{l}c_{n_{1}+1}^{\dagger} c_{n_{1}+n_{2}+2}^{\dagger} \ldots c_{n_{1}+\ldots+n_{p-1}+p-1}^{\dagger}\left|\Omega_{F}\right\rangle, \\ 0, \quad n_{1}+\ldots+n_{p} \neq q\end{array}\right.$

$n_{1}+\ldots+n_{p}=q$

where the pseudovacuum state $\left|\Omega_{F}\right\rangle$ is annihilated by all $c_{A}$ 's.

\section{Appendix B. More on Yang-Mills theory on punctured surfaces}

Let us discuss the analogue of the Yang-Mills connections in the parabolic setting. We want to extremize the Yang-Mills action together with the action of the sources coming from the Wilson points:

$$
-S_{\mathrm{ym}}=\mathrm{i} \int_{\Sigma_{g}} \operatorname{Tr}\left(\sigma F_{A}\right)+\frac{g_{\mathrm{ym}}^{2}}{2} \int_{\Sigma} \operatorname{Tr} \sigma^{2}+\mathrm{i} \sum_{i=1}^{n} \operatorname{Tr} \sigma\left(x_{i}\right) J_{i}
$$

so that the path integral measure was $e^{-S_{\text {ym }}}$, where $J_{i} \in O_{v_{i}}$ is allowed to vary as well. Varying w.r.t $J_{i}$ we get: $J_{i}$ is one of the fixed points of the $G$-action on $O_{v_{i}}$ generated by $\sigma\left(x_{i}\right)$, while

$$
\begin{aligned}
& F_{A}+\sum_{i} J_{i} \delta^{(2)}\left(x_{i}\right)=\mathrm{i} g_{\mathrm{ym}}^{2} \sigma \\
& d_{A} \sigma=0
\end{aligned}
$$


the equations, for generic non-zero $\sigma$, imply that the structure group $G$ of the bundle reduces to $T$, its maximal torus, $\sigma, A, J_{i} \in \mathrm{t}$, moreover: $\sigma=$ const, $F_{A}=f+d a, a \in \Omega^{1}\left(\Sigma_{g}\right) \otimes \mathrm{t}$ and $f$ is a harmonic two-form on $\Sigma_{g}$ valued in $\mathrm{t}$, moreover

$$
\int_{\Sigma_{g}} f \in 2 \pi \Lambda_{\mathrm{cw}}=\operatorname{Hom}(U(1), G)
$$

Now, 175 implies, for abelian $A, f, a, J_{i}$

$$
\int_{\Sigma_{g}} f+\sum_{i=1}^{n} J_{i}=-\mathrm{i} g_{\mathrm{ym}}^{2} A_{\Sigma_{g}} \sigma
$$

which means that $\sigma$ takes quantized values as in the unramified case, but the lattice is shifted by the amount which depends on $J_{i}$ 's. Moreover, the choices of $J_{i}$ 's are labelled by the cosets $W / W_{v_{i}}, i=1, \ldots, n$, so we get a multitude of lattices labelled by

$$
\searrow_{i=1}^{n} W / W_{v_{i}}
$$

Moreover, the overall $W$-action is a symmetry, so finally, the set of critical points is the quotient

$$
\left(\Lambda_{\mathrm{cW}} \times \searrow_{i=1}^{n} W / W_{v_{i}}\right) / W
$$

Let us make an explicit calculation for $G=S U(2)$. The orbits $O_{v_{i}} \approx \mathbb{C P}^{1}$ for non-zero $v_{i}$ 's, and $W=\mathbb{Z}_{2}$. Let us assume the eigenvalues $\pm \mathrm{i} v_{i}$ of the traceless two by two antihermitian matrices $J_{i}, i=1, \ldots, n$ are in generic position. Let the metric on $\Sigma_{g}$ be

$$
d s^{2}=\mathrm{g}_{z \bar{z}} d z d \bar{z}, \quad \frac{1}{2 \mathrm{i}} \int_{\Sigma_{g}} \mathrm{~g}_{z \bar{z}} d z \wedge d \bar{z}=A_{\Sigma_{g}}
$$

Then, the generalized Yang-Mills connection has $f=m \frac{\pi}{A_{\Sigma_{g}}} \mathrm{~g}_{z \bar{z}} d z \wedge d \bar{z}, m \in \mathbb{Z}$,

$$
\sigma=-\frac{1}{g_{\mathrm{ym}}^{2} A_{\Sigma_{g}}}\left(2 \pi m+\sum_{i=1}^{n} \pm v_{i}\right)
$$

and $a=\mathrm{i} \star d \varphi$ with

$$
\Delta \varphi=-\sum_{i=1}^{n} \pm v_{i}\left(\delta^{(2)}\left(z-x_{i}\right)-\frac{1}{A_{\Sigma_{g}}}\right)
$$

Despite the appearences, there is no singularity in the value of the action:

$$
-S_{\mathrm{ym}}=-g_{\mathrm{ym}}^{2} A_{S^{2}} \sigma^{2}=-\frac{4 \pi^{2}}{g_{\mathrm{ym}}^{2} A_{\Sigma_{g}}}\left(m+\sum_{i=1}^{n} \pm \frac{v_{i}}{2 \pi}\right)^{2}
$$


Cf. the partition function:

$Z\left(g_{\mathrm{ym}}^{2} A_{\Sigma_{g}} ; \boldsymbol{\nu}\right)=\frac{1}{(2 \pi \mathrm{i})^{n}} \sum_{\tilde{m} \in \mathbb{Z}} \frac{e^{-g_{\mathrm{ym}}^{2} A_{\Sigma_{g}} \tilde{m}^{2}}}{\tilde{m}^{n-2+2 g}} \prod_{i=1}^{n}\left(e^{\mathrm{i} v_{i} \tilde{m}}-e^{-\mathrm{i} v_{i} \tilde{m}}\right)=-\frac{1}{\pi^{2}} \sum_{e \in 2^{n-1}}(-1)^{e} \gamma_{n-2+2 g}\left(\nu_{e} ; g_{\mathrm{ym}}^{2} A_{\Sigma_{g}}\right)$

where $2^{n-1}$ is the set of $n$-tuples of \pm 1 , up to the overall change of sign, $\nu_{e}=\sum_{i=1}^{n} \pm v_{i}$, $(-1)^{e}=\prod_{i=1}^{n} \pm 1$

$$
\gamma_{k}(x ; t)= \begin{cases}\sum_{\tilde{m}=1}^{\infty} \frac{\cos (\tilde{m} x)}{(2 \pi \mathrm{i} \tilde{m})^{k}} e^{-t \tilde{m}^{2}}, \quad k-\text { even } \\ \sum_{\tilde{m}=1}^{\infty} \frac{\sin (\tilde{m} x)}{(2 \pi \mathrm{i} \tilde{m})^{k}} e^{-t \tilde{m}^{2}}, \quad k-\text { odd }\end{cases}
$$

Now,

$$
\left(4 \pi^{2} \partial_{t}\right)^{l} \gamma_{2 l}(x ; t)=-\frac{1}{2}+\frac{1}{2} \sum_{\tilde{m} \in \mathbb{Z}} e^{\mathrm{i} \tilde{m} x-t \tilde{m}^{2}}=-\frac{1}{2}+\frac{1}{\sqrt{8 \pi t}} \sum_{n \in \mathbb{Z}} e^{-\frac{(x+2 \pi n)^{2}}{4 t}}
$$

which means that as long as $x \notin 2 \pi \mathbb{Z}, \gamma_{2 l}(x ; t)$, for small $t>0$ behaves as a polynomial of degree $t$ plus exponentially small corrections. Similarly

$$
\left(2 \pi \mathrm{i} \partial_{x}\right)\left(4 \pi^{2} \partial_{t}\right)^{l} \gamma_{2 l+1}(x ; t)=-\frac{1}{2}+\frac{1}{\sqrt{8 \pi t}} \sum_{n \in \mathbb{Z}} e^{-\frac{(x+2 \pi n)^{2}}{4 t}}
$$

Finally, the heat equation

$$
\left(\left(2 \pi \mathrm{i} \partial_{x}\right)^{2}+\partial_{t}\right) \gamma_{k}(x ; t)=0
$$

relates the $t$ and the $x$ expansion for small $t$ and $x$.

The small $g^{2} A_{S^{2}}$ expansion of (181) can be done using the following elementary function:

$$
\begin{aligned}
\sum_{\tilde{m}=1}^{\infty} \frac{\cos (\tilde{m} \theta)}{\tilde{m}^{2}}=\frac{\pi^{2}}{6}-\frac{\theta(2 \pi-\theta)}{4}, \quad \text { for } \quad 0 \leq \theta \leq & 2 \pi \\
& =-\frac{\pi^{2}}{12}-\min _{n \in \mathbb{Z}} \frac{(\theta-(2 n+1) \pi)^{2}}{4}
\end{aligned}
$$

which gives, for $n=4$ :

$$
\begin{aligned}
& Z\left(g^{2} A_{S^{2}} ; v_{1}, v_{2}, v_{3}, v_{4}\right)= \\
& \quad \frac{1}{(2 \pi \mathrm{i})^{n}} \sum_{\tilde{m} \in \mathbb{Z}} \frac{e^{-g^{2} A_{S^{2}} \tilde{m}^{2}}}{\tilde{m}^{2}} \prod_{i=1}^{n}\left(e^{\mathrm{i} v_{i} \tilde{m}}-e^{-\mathrm{i} v_{i} \tilde{m}}\right)=-\frac{1}{2 \pi^{2}} \sum_{e \in 2^{n}}(-1)^{e} \gamma_{n-2}\left(\nu_{e} ; g^{2} A_{S^{2}}\right)
\end{aligned}
$$

The piece-wise polynomial dependence on $v_{i}$ 's is a consequence of the Duistermaat-Heckman's theorem [36]. Indeed, we can view $v_{i}$ 's as the levels of the abelian moment maps by writing

$$
J_{i}=I_{i} I_{i}^{\dagger}-\frac{1}{2} I_{i}^{\dagger} I_{i} \mathbf{1}_{2}
$$

with

$$
I_{i}^{\dagger} I_{i}=v_{i}
$$

being the $U(1)_{i}$ moment map. 


\section{References}

[1] M. Aganagic and A. Okounkov, Quasimap counts and Bethe eigenfunctions, arXiv:1704.08746 [math-ph].

[2] L. F. Alday, D. Gaiotto and Y. Tachikawa, Liouville Correlation Functions from Four-dimensional Gauge Theories, Lett. Math. Phys. 91, 167 (2010) arXiv:0906.3219 [hep-th]].

[3] L. F. Alday, D. Gaiotto, S. Gukov, Y. Tachikawa and H. Verlinde, Loop and surface operators in $\mathrm{N}=2$ gauge theory and Liouville modular geometry, JHEP 1001, 113 (2010) doi:10.1007/JHEP01(2010)113 arXiv:0909.0945 [hep-th]].

[4] L. F. Alday and Y. Tachikawa, Affine SL(2) conformal blocks from 4d gauge theories, Lett. Math. Phys. 94, 87 (2010) doi:10.1007/s11005-010-0422-4 arXiv:1005.4469 [hep-th]].

[5] G. Arutyunov, S. Frolov and P. Medvedev, Elliptic Ruijsenaars-Schneider model from the cotangent bundle over the two-dimensional current group, Journal of Mathematical Physics 38 (1997) 5682

[6] S. K. Ashok, M. Billo, E. Dell'Aquila, M. Frau, V. Gupta, R. R. John and A. Lerda, Surface operators, chiral rings, and localization in $\mathcal{N}=2$ gauge theories, JHEP 1711, 137 (2017) doi:10.1007/JHEP11(2017)137 [arXiv:1707.08922 [hep-th]].

[7] H. Awata, A. Tsuchiya, Y. Yamada, Integral formulas for the WZNW correlation functions, Nucl. Phys., B365 (1991), 680-698.

[8] H. Awata, H. Fuji, H. Kanno, M. Manabe and Y. Yamada, Localization with a Surface Operator, Irregular Conformal Blocks and Open Topological String, Adv. Theor. Math. Phys. 16, no. 3, 725 (2012) doi:10.4310/ATMP.2012.v16.n3.a1 arXiv:1008.0574 [hep-th]].

[9] H. M. Babujian, Off-shell Bethe Ansatz equation and N point correlators in $\mathrm{SU}(2)$ WZNW theory, J. Phys. A 26, 6981 (1993) doi:10.1088/0305-4470/26/23/037 |hepth/9307062.

[10] H. M. Babujian and R. Flume, Off-shell Bethe Ansatz equation for Gaudin magnets and solutions of Knizhnik-Zamolodchikov equations, Mod. Phys. Lett. A 9, 2029 (1994) doi:10.1142/S0217732394001891 [hep-th/9310110].

[11] A. Barns-Graham, N. Dorey, N. Lohitsiri, D. Tong and C. Turner, ADHM and the 4d Quantum Hall Effect, arXiv:1710.09833 [hep-th].

[12] L. Baulieu, A. Losev and N. Nekrasov, Chern-Simons and twisted supersymmetry in various dimensions, Nucl. Phys. B 522, 82 (1998) doi:10.1016/S05503213(98)00096-0 [hep-th/9707174].

[13] M. Bershadsky, A. Johansen, V. Sadov and C. Vafa, Topological reduction of 4-d SYM to 2-d sigma models, Nucl. Phys. B 448, 166 (1995) doi:10.1016/05503213(95)00242-K [hep-th/9501096. 
[14] A. Bertram, I. Ciocan-Fontanine and B. s. Kim, Two proofs of a conjecture of Hori and Vafa, math/0304403 [math-ag].

[15] O. Biquard, Sur les Fibrés Paraboliques sur une Surface Complexe, J. London Math. Soc. 53 (1996) 302.

[16] I. Biswas, Parabolic bundles as obrifold bundles, Duke Math J. 88 (1997), no. 2, 305-325

[17] M. Blau and G. Thompson, Derivation of the Verlinde formula from Chern-Simons theory and the G/G model, Nucl. Phys. B 408, 345 (1993) doi:10.1016/05503213(93)90538-Z |hep-th/9305010].

[18] M. Blau and G. Thompson, Lectures on 2-d gauge theories: Topological aspects and path integral techniques, hep-th/9310144.

[19] A. Braverman, Instanton counting via affine Lie algebras. 1. Equivariant J-functions of (affine) flag manifolds and Whittaker vectors, math/0401409 [math$\mathrm{ag}]$.

[20] A. Braverman, D. Maulik and A. Okounkov, Quantum cohomology of the Springer resolution, arXiv:1001.0056 [math.AG].

[21] U. Bruzzo, W.-y. Chuang, D.-E. Diaconescu, M. Jardim, G. Pan and Y. Zhang, D-branes, surface operators, and ADHM quiver representations, Adv. Theor. Math. Phys. 15, no. 3, 849 (2011) doi:10.4310/ATMP.2011.v15.n3.a6 arXiv:1012.1826 [hep-th]].

[22] M. Bullimore, H. C. Kim and P. Koroteev, Defects and Quantum Seiberg-Witten Geometry, JHEP 1505, 095 (2015) doi:10.1007/JHEP05(2015)095 arXiv:1412.6081 [hep-th]].

[23] M. Bullimore, H. C. Kim and T. Lukowski, Expanding the Bethe/Gauge Dictionary, JHEP 1711, 055 (2017) doi:10.1007/JHEP11(2017)055

[24] C. K. Chang, H. Y. Chen, D. Jain and N. Lee, Connecting Localization and Wall-Crossing via D-Branes, arXiv:1512.02645 [hep-th].

[25] H. Y. Chen, N. Dorey, T. J. Hollowood and S. Lee, A New 2d/4d Duality via Integrability, JHEP 1109, 040 (2011) doi:10.1007/JHEP09(2011)040 arXiv:1104.3021 [hep-th]].

[26] K. Costello, Supersymmetric gauge theory and the Yangian, arXiv:1303.2632 [hepth].

[27] K. Costello, Integrable lattice models from four-dimensional field theories, Proc. Symp. Pure Math. 88, 3 (2014) doi:10.1090/pspum/088/01483 arXiv:1308.0370 [hep-th]].

[28] L. Dixon, D. Friedan, E. Martinec and S. Shenker, The Conformal Field Theory of Orbifolds, Nucl. Phys. B 282, 13-73 (1987). 
[29] V. S. Dotsenko and V. A. Fateev, Conformal Algebra and Multipoint Correlation Functions in Two-Dimensional Statistical Models, Nucl. Phys. B 240, 312 (1984). doi:10.1016/0550-3213(84)90269-4

[30] M. R. Douglas, Branes within branes, in Cargese 1997, Strings, branes and dualities, 267-275 [hep-th/9512077].

[31] M. R. Douglas, Gauge fields and D-branes, J. Geom. Phys. 28, 255 (1998) |hepth/9604198.

[32] M. R. Douglas and G. W. Moore, D-branes, quivers, and ALE instantons, hepth/9603167.

[33] N. Dorey, S. Lee and T. J. Hollowood, Quantization of Integrable Systems and a 2d/4d Duality, JHEP 1110, 077 (2011) doi:10.1007/JHEP10(2011)077 arXiv:1103.5726 [hep-th]].

[34] N. Dorey and P. Zhao, Solution of quantum integrable systems from quiver gauge theories, JHEP 1702, 118 (2017) doi:10.1007/JHEP02(2017)118 arXiv:1512.09367 [hep-th]].

[35] V. G. Drinfeld, Sov. Math. Dokl. 36 (1987) 212-216.

[36] J. J. Duistermaat and G. J. Heckman, On The Variation In The Cohomology In The Symplectic Form Of The Reduced Phase Space Invent. Math. 69 (1982) 259.

[37] M. Finkelberg, A. Kuznetsov, Parabolic sheaves on surfaces and affine Lie algebra $\hat{\mathfrak{g l}}_{n}$, J. Reine Angew. Math. 529 (2000), 155-203.

[38] V. Fock, A. Rosly, Poisson structure on moduli of flat connections on Riemann surfaces and $r$-matrix, Am. Math. Soc. Transl. 191 (1999) 67-86

[39] E. Frenkel, Free field realizations in representation theory and conformal field theory, hep-th/9408109.

[40] E. Frenkel, S. Gukov and J. Teschner, Surface Operators and Separation of Variables, JHEP 1601, 179 (2016) doi:10.1007/JHEP01(2016)179 arXiv:1506.07508 [hep-th]].

[41] E. Frenkel, N. Reshetikhin, The q-characters of representations of quantun affine algebras and deformations of $W$-algebras, arXiv:math/9810055v5 [math.QA]

[42] I. B. Frenkel and V. G. Kac, Basic Representations of affine Lie algebras and dual resonance models, Inv. Math. 62, 23 (1980).

[43] D. Gaiotto, N=2 dualities, JHEP 1208, 034 (2012) arXiv:0904.2715 [hep-th]].

[44] D. Gaiotto, Surface Operators in N=2 4d Gauge Theories, JHEP 1211, 090 (2012) doi:10.1007/JHEP11(2012)090 [arXiv:0911.1316 [hep-th]].

[45] D. Gaiotto, S. Gukov and N. Seiberg, Surface Defects and Resolvents, JHEP 1309, 070 (2013) doi:10.1007/JHEP09(2013)070 arXiv:1307.2578 [hep-th]]. 
[46] D. Gaiotto, G. W. Moore and A. Neitzke, Framed BPS States, Adv. Theor. Math. Phys. 17, no. 2, 241 (2013) doi:10.4310/ATMP.2013.v17.n2.a1 arXiv:1006.0146 [hep-th]].

[47] O. Gamayun, N. Iorgov and O. Lisovyy, Conformal field theory of Painlevé VI, JHEP 1210, 038 (2012) Erratum: [JHEP 1210, 183 (2012)] doi:10.1007/JHEP10(2012)183, 10.1007/JHEP10(2012)038 |arXiv:1207.0787 [hepth]].

[48] A. Gerasimov, A. Morozov, M. Olshanetsky, A. Marshakov and S. L. Shatashvili, Wess-Zumino-Witten model as a theory of free fields, Int. J. Mod. Phys. A 5, 2495 (1990) (originally published as 4 preprints in April 1989) doi:10.1142/S0217751X9000115X

[49] A. Gerasimov, Localization in GWZW and Verlinde formula, hep-th/9305090.

[50] A. A. Gerasimov and S. L. Shatashvili, On exact tachyon potential in open string field theory, JHEP 0010, 034 (2000) hep-th/0009103.

[51] A. A. Gerasimov and S. L. Shatashvili, Higgs Bundles, Gauge Theories and Quantum Groups, Commun. Math. Phys. 277, 323 (2008) doi:10.1007/s00220007-0369-1 hep-th/0609024.

[52] A. A. Gerasimov and S. L. Shatashvili, Two-dimensional gauge theories and quantum integrable systems, Proceedings of Symposia in Pure Mathematics, May 25-29 2007, University of Augsburg, Germany |arXiv:0711.1472 [hep-th]].

[53] A. Givental, Equivariant Gromov - Witten Invariants, arXiv:alg-geom/9603021

[54] A. Givental, A mirror theorem for toric complete intersections, arXiv:alggeom/9701016v2

[55] A. Givental, The mirror formula for quintic threefolds, arXiv:math/9807070

[56] J. Gomis and B. Le Floch, M2-brane surface operators and gauge theory dualities in Toda, JHEP 1604, 183 (2016) doi:10.1007/JHEP04(2016)183 arXiv:1407.1852 [hep-th]].

[57] J. Gomis, T. Okuda and V. Pestun, Exact Results for 't Hooft Loops in Gauge Theories on $\mathrm{S}^{\wedge} 4$, JHEP 1205, $14 \overline{\text { (2012) doi:10.1007/JHEP05(2012)141 }}$ arXiv:1105.2568 [hep-th]].

[58] A. Gorsky, B. Le Floch, A. Milekhin and N. Sopenko, Surface defects and instantonĐvortex interaction, Nucl. Phys. B 920, 122 (2017) doi:10.1016/j.nuclphysb.2017.04.010 [arXiv:1702.03330 [hep-th]].

[59] A. Gorsky and N. Nekrasov, Hamiltonian systems of Calogero type and Two dimensional Yang-Mills theory, Nucl. Phys. B414 (1994) 213-238, arXiv:hepth/9304047 
[60] A. Gorsky and N. Nekrasov, Relativistic Calogero-Moser system as gauged WZW theory, Nucl. Phys. B436 (1995) 582-608, arXiv:hep-th/9401017

[61] A. Gorsky and N. Nekrasov, Elliptic Calogero-Moser system from two dimensional current algebra, arXiv:hep-th/9401021

[62] S. Gukov, Gauge theory and knot homologies, Fortsch. Phys. 55, 473 (2007) doi:10.1002/prop.200610385 [arXiv:0706.2369 [hep-th]].

[63] S. Gukov and E. Witten, Rigid Surface Operators, Adv. Theor. Math. Phys. 14, no. 1, 87 (2010) doi:10.4310/ATMP.2010.v14.n1.a3 [arXiv:0804.1561 [hep-th]].

[64] Y. Ito, T. Okuda and M. Taki, Line operators on $\mathrm{S}^{\wedge} 1 \mathrm{xR} \wedge 3$ and quantization of the Hitchin moduli space, JHEP 1204, 010 (2012) Erratum: [JHEP 1603, 085 (2016)] doi:10.1007/JHEP03(2016)085, 10.1007/JHEP04(2012)010 arXiv:1111.4221 [hepth]].

[65] K. Hosomichi, S. Lee and T. Okuda, Supersymmetric vortex defects in two dimensions, arXiv:1705.10623 [hep-th].

[66] K. Hori and C. Vafa, Mirror symmetry, hep-th/0002222.

[67] S. Jeong and N. Nekrasov, Opers, surface defects, and Yang-Yang functional, arXiv:1806.08270 [hep-th].

[68] H. Kanno and Y. Tachikawa, Instanton counting with a surface operator and the chain-saw quiver, JHEP 1106, 119 (2011) arXiv:1105.0357 [hep-th]].

[69] S. Katz, A. Klemm and C. Vafa, Geometric engineering of quantum field theories, Nucl. Phys. B 497, 173 (1997) hep-th/9609239.

[70] A. King, Instantons and holomorphic bundles on the blown up plane, Ph.D. thesis, Oxford, 1989.

[71] P. Koroteev, P. P. Pushkar, A. Smirnov and A. M. Zeitlin, Quantum K-theory of Quiver Varieties and Many-Body Systems, arXiv:1705.10419 [math.AG].

[72] P. Koroteev and A. M. Zeitlin, Difference Equations for K-theoretic Vertex Functions of Type-A Nakajima Varieties, arXiv:1802.04463 [math.AG].

[73] P. Kronheimer and T. Mrowka, Gauge theory for embedded surfaces, I, Topology 32 (1993) 773.

[74] P. Kronheimer and T. Mrowka, Gauge theory for embedded surfaces, II, Topology 34 (1995) 37.

[75] C. Kozcaz, S. Pasquetti, F. Passerini and N. Wyllard, Affine sl(N) conformal blocks from $\mathrm{N}=2 \mathrm{SU}(\mathrm{N})$ gauge theories, JHEP 1101, 045 (2011) doi:10.1007/JHEP01(2011)045 [arXiv:1008.1412 [hep-th]].

[76] P. Kronheimer, and H. Nakajima, Yang-Mills instantons on ALE gravitational instantons, Math. Ann. 288 (1990) 263-307. 
[77] A. Losev, A. Marshakov and N. A. Nekrasov, Small instantons, little strings and free fermions, In *Shifman, M. (ed.) et al.: From fields to strings, vol. 1* 581-621 hep-th/0302191.

[78] A. Losev, G. W. Moore, N. Nekrasov and S. Shatashvili, Nucl. Phys. Proc. Suppl. 46, 130 (1996) doi:10.1016/0920-5632(96)00015-1 [hep-th/9509151.

[79] A. Losev, N. Nekrasov and S. L. Shatashvili, Testing Seiberg-Witten solution, In *Cargese 1997, Strings, branes and dualities* 359-372 [hep-th/9801061].

[80] A. Losev, N. Nekrasov and S. L. Shatashvili, The Freckled instantons, In *Shifman, M.A. (ed.): The many faces of the superworld* 453-475 doi:10.1142/9789812793850_0026 |hep-th/9908204].

[81] A. Losev, N. Nekrasov and S. L. Shatashvili, Freckled instantons in two-dimensions and four-dimensions, Class. Quant. Grav. 17, 1181 (2000) doi:10.1088/02649381/17/5/327 [hep-th/9911099].

[82] D. Maulik and A. Okounkov, Quantum Groups and Quantum Cohomology, arXiv:1211.1287 [math.AG].

[83] G. W. Moore, N. Nekrasov and S. Shatashvili, Integrating over Higgs branches, Commun. Math. Phys. 209, 97 (2000) hep-th/9712241.

[84] H. Nakajima, Instantons on ALE spaces, quiver varieties, and Kac-Moody algebras, Duke Math. J. 76 (1994), no. 2, 365-416

[85] H. Nakajima, Instantons and affine Lie algebras, Nucl. Phys. B-Proceedings Suppl. 46 (1996) 1-3, 154-161

[86] H. Nakajima, Quiver varieties and Kac-Moody algebras, Duke Math. J. 91 (1998), no. 3, 515-560. doi:10.1215/S0012-7094-98-09120-7

[87] H. Nakajima and K. Yoshioka, Instanton counting on blowup. 1., Invent. Math. 162, 313 (2005) doi:10.1007/s00222-005-0444-1 |math/0306198 [math.AG]].

[88] H. Nakajima and K. Yoshioka, Perverse coherent sheaves on blow-up. I. A Quiver description, arXiv:0802.3120 [math.AG].

[89] H. Nakajima and K. Yoshioka, Perverse coherent sheaves on blowup, III: Blow-up formula from wall-crossing, Kyoto J. Math. 51, no. 2, 263 (2011) doi:10.1215/21562261-1214366 [arXiv:0911.1773 [math.AG]].

[90] S. Nawata, Givental J-functions, Quantum integrable systems, AGT relation with surface operator, Adv. Theor. Math. Phys. 19, 1277 (2015) doi:10.4310/ATMP.2015.v19.n6.a4 arXiv:1408.4132 [hep-th]].

[91] N. Nekrasov, Four dimensional holomorphic theories, PhD thesis, Princeton University, 1996, available from http://scgp.stonybrook.edu/people/faculty/bios/nikita-nekrasov 
[92] N. Nekrasov, Holomorphic bundles and integrable systems, Commun.Math.Phys. 180 (1996) 587-604 arXiv:hep-th/9503157

[93] N. Nekrasov, On the BPS/CFT correspondence, Lecture at the University of Amsterdam string theory group seminar (Feb. 3, 2004).

[94] N. A. Nekrasov, Seiberg-Witten prepotential from instanton counting, Adv. Theor. Math. Phys. 7, no. 5, 831 (2003) [hep-th/0206161].

[95] N. Nekrasov, A. Rosly and S. Shatashvili, Darboux coordinates, Yang-Yang functional, and gauge theory, Nucl. Phys. Proc. Suppl. 216, 69 (2011) doi:10.1016/j.nuclphysbps.2011.04.150 arXiv:1103.3919 [hep-th]].

[96] N. Nekrasov, BPS/CFT correspondence: non-perturbative Dyson-Schwinger equations and qq-characters, JHEP 1603, 181 (2016) arXiv:1512.05388 [hep-th].

[97] N. Nekrasov, BPS/CFT correspondence II: Instantons at crossroads, Moduli and Compactness Theorem, arXiv:1608.07272 [hep-th].

[98] N. Nekrasov, BPS/CFT correspondence III: Gauge Origami Partition Function and qq-characters, arXiv:1701.00189 [hep-th].

[99] N. Nekrasov, BPS/CFT correspondence V: BPZ and KZ equations from qq-characters, arXiv:1711.11582 [hep-th].

[100] N. Nekrasov, Bethe States As Defects In Gauge Theories, Bethe wavefunctions from gauged linear sigma models via Bethe/gauge correspondence, talks at the SCGP, delivered on 2013-10-02 and 2014-1103, http://scgp.stonybrook.edu/video_portal/video.php?id=1360

[101] N. Nekrasov, Supersymmetric gauge theories and quantization of integrable systems, lecture at Strings'2009, Rome, http://strings2009.roma2.infn.it/talks/Nekrasov_Strings09.pdf

[102] N. Nekrasov and A. Okounkov, Seiberg-Witten theory and random partitions, Prog. Math. 244, 525 (2006) hep-th/0306238.

[103] N. Nekrasov and V. Pestun, Seiberg-Witten geometry of four dimensional N=2 quiver gauge theories, arXiv:1211.2240 [hep-th].

[104] N. Nekrasov, V. Pestun and S. Shatashvili, Quantum geometry and quiver gauge theories, arXiv:1312.6689 [hep-th].

[105] N. Nekrasov and N. S. Prabhakar, Spiked Instantons from Intersecting D-branes, Nucl. Phys. B 914, 257 (2017) doi:10.1016/j.nuclphysb.2016.11.014 arXiv:1611.03478 [hep-th].

[106] N. A. Nekrasov and S. L. Shatashvili, Supersymmetric vacua and Bethe ansatz, Nucl. Phys. Proc. Suppl. 192-193, 91 (2009) doi:10.1016/j.nuclphysbps.2009.07.047 arXiv:0901.4744 [hep-th]. 
[107] N. A. Nekrasov and S. L. Shatashvili, Quantum integrability and supersymmetric vacua, Prog. Theor. Phys. Suppl. 177, 105 (2009) doi:10.1143/PTPS.177.105 arXiv:0901.4748 [hep-th].

[108] N. A. Nekrasov and S. L. Shatashvili, Quantization of Integrable Systems and Four Dimensional Gauge Theories, arXiv:0908.4052 [hep-th].

[109] A. Okounkov and A. Smirnov, Quantum difference equation for Nakajima varieties, arXiv:1602.09007 [math-ph].

[110] Y. Pan and W. Peelaers, Intersecting Surface Defects and Instanton Partition Functions, JHEP 1707, 073 (2017) doi:10.1007/JHEP07(2017)073 arXiv:1612.04839[hep-th]].

[111] R. Poghossian, Deformed SW curve and the null vector decoupling equation in Toda field theory, JHEP 1604, 070 (2016) doi:10.1007/JHEP04(2016)070 arXiv:1601.05096 [hep-th]].

[112] G. Poghosyan and R. Poghossian, VEV of Baxter's Q-operator in $\mathrm{N}=2$ gauge theory and the BPZ differential equation, JHEP 1611, 058 (2016) doi:10.1007/JHEP11(2016)058 [arXiv:1602.02772 [hep-th]].

[113] G. Segal, Unitary representations of some infinite-dimensional groups, Commun. Math. Phys. Vol. 80, No. 3, 301-342, (1981).

[114] N. Seiberg and E. Witten, Electric - magnetic duality, monopole condensation, and confinement in $\mathrm{N}=2$ supersymmetric Yang-Mills theory, Nucl. Phys. B 426, 19 (1994) Erratum: [Nucl. Phys. B 430, 485 (1994)] |hep-th/9407087].

[115] N. Seiberg and E. Witten, Monopoles, duality and chiral symmetry breaking in $\mathrm{N}=2$ supersymmetric QCD, Nucl. Phys. B 431, 484 (1994) [hep-th/9408099].

[116] N. Seiberg and E. Witten, String theory and noncommutative geometry, JHEP 9909, 032 (1999) hep-th/9908142.

[117] M. Shifman and A. Yung, Quantum Deformation of the Effective Theory on Non-Abelian string and 2D-4D correspondence, Phys. Rev. D 89, no. 6, 065035 (2014) doi:10.1103/PhysRevD.89.065035 [arXiv:1401.1455 [hep-th]].

[118] V. Tarasov and A. Varchenko, Jackson integral representations for solutions of the quantized Knizhnik-Zamolodchikov equation, [hep-th/9311040.

[119] E. P. Verlinde, Fusion Rules and Modular Transformations in 2D Conformal Field Theory, Nucl. Phys. B 300, 360 (1988).

[120] E. Witten, Two-dimensional gauge theory revisited, J. Geom. Phys. 9 (1992), 303368

[121] E. Witten, Phases of $\mathrm{N}=2$ theories in two dimensions, Nucl. Phys. B 403, 159 (1993) hep-th/9301042. 
[122] E. Witten, Supersymmetric Yang-Mills theory on a four manifold, J. Math. Phys. 35, 5101 (1994) doi:10.1063/1.530745 [hep-th/9403195].

[123] E. Witten, Solutions of four-dimensional field theories via M theory, Nucl. Phys. B 500, 3 (1997) [hep-th/9703166].

[124] E. Witten, Integrable Lattice Models From Gauge Theory, arXiv:1611.00592 [hepth].

[125] E. Witten, The Verlinde algebra and the cohomology of the Grassmannian, In *Cambridge 1993, Geometry, topology, and physics* 357-422 [hep-th/9312104]. 